\title{
LOCAL ANALYSIS FOR SEMI-BOUNDED GROUPS
}

\author{
PANTELIS E. ELEFTHERIOU
}

\begin{abstract}
An o-minimal expansion $\mathcal{M}=\langle M,<,+, 0, \ldots\rangle$ of an ordered group is called semi-bounded if it does not expand a real closed field. Possibly, it defines a real closed field with bounded domain $I \subseteq M$. Let us call a definable set short if it is in definable bijection with a definable subset of some $I^{n}$, and long otherwise. Previous work by Edmundo and Peterzil provided structure theorems for definable sets with respect to the dichotomy 'bounded versus unbounded'. In [Pet3], Peterzil conjectured a refined structure theorem with respect to the dichotomy 'short versus long'. In this paper, we prove Peterzil's conjecture. In particular, we obtain a quantifier elimination result down to suitable existential formulas in the spirit of [vdD1]. Furthermore, we introduce a new closure operator that defines a pregeometry and gives rise to the refined notions of 'long dimension' and 'long-generic' elements. Those are in turn used in a local analysis for a semi-bounded group $G$, yielding the following result: on a long direction around each long-generic element of $G$ the group operation is locally isomorphic to $\left\langle M^{k},+\right\rangle$.
\end{abstract}

\section{INTRODUCTION}

For an o-minimal expansion $\mathcal{M}=\langle M,<,+, 0, \ldots\rangle$ of an ordered group, there are naturally three possibilities: $\mathcal{M}$ is either (a) linear, (b) semi-bounded (and non-linear), or (c) it expands a real closed field. Let us define the first two.

Definition 1.1. Let $\Lambda$ be the set of all partial $\emptyset$-definable endomorphisms of $\langle M,<$ $,+, 0\rangle$, and $\mathcal{B}$ the collection of all bounded definable sets. Then $\mathcal{M}$ is called linear ([LP]) if every definable set is already definable in $\left\langle M,<,+, 0,\{\lambda\}_{\lambda \in \Lambda}\right\rangle$, and it is called semi-bounded ([Ed, Pet1]) if every definable set is already definable in $\left\langle M,<,+, 0,\{\lambda\}_{\lambda \in \Lambda},\{B\}_{B \in \mathcal{B}}\right\rangle$.

Obviously, if $\mathcal{M}$ is linear then it is semi-bounded. By [PeSt], $\mathcal{M}$ is not linear if and only if there is a real closed field defined on some bounded interval. By [Ed], $\mathcal{M}$ is not semi-bounded if and only if $M$ expands a real closed field if and only if for any two intervals there is a definable bijection between them.

It is largely evident from the literature that among the three cases, (a) and (c) have provided the most accommodating settings for studying general mathematics. For example, the definable sets in a real closed field are the main objects of study in semialgebraic geometry (a classical reference is [DK]). Moreover, o-minimal linear topology naturally extends the classical subject of piecewise linear topology

Date: March 10, 2010.

2000 Mathematics Subject Classification. 03C64.

Key words and phrases. O-minimality, semi-bounded structures, definable groups, pregeometries.

Research supported by the Fundação para a Ciência e a Tecnologia grants SFRH/BPD/35000/2007 and PTDC/MAT/101740/2008. 
and has the potential to tackle problems that arise in the study of algebraically closed valued fields (see, for example, [HL]). From an internal aspect, the study of definable groups in both of these two settings has been rather successful (see further comments below).

On the other hand, the middle case (b) remains as elusive as interesting from a classification point of view. Although a local field may be definable, and thus the definable structure can get quite rich, there is no global field, and hence many known technics do not apply. In particular, little is known with respect to structure theorems of definable groups in this setting. In this paper, we set forth a project of analyzing semi-bounded groups, mainly motivated by two conjectures asked by Peterzil in [Pet3]. Let us describe our project.

For the rest of the paper, we fix a semi-bounded o-minimal expansion $\mathcal{M}=\langle M,<,+, 0, \ldots\rangle$ of an ordered group, which is not linear. We fix an element $1>0$ such that a real closed field, whose universe is $(0,1)$ and whose order agrees with $<$, is definable in $\mathcal{M}$.

Let $\mathcal{L}$ denote the underlying language of $\mathcal{M}$. By 'definable' we mean 'definable in $\mathcal{M}^{\prime}$ possibly with parameters. A group $G$ is said to be definable if both its domain and its group operation are definable. Definable sets and groups in this setting are also referred to as semi-bounded. If they are defined in the linear reduct $\mathcal{M}_{\text {lin }}=\left\langle M,<,+, 0,\{\lambda\}_{\lambda \in \Lambda}\right\rangle$ of $\mathcal{M}$, we call them semi-linear. The underlying language of $\mathcal{M}_{\text {lin }}$ is denoted by $\mathcal{L}_{\text {lin }}$.

An interval $I \subseteq M$ is called short if there is a definable bijection between $I$ and $(0,1)$; otherwise, it is called long. An element $a \in M$ is called short if either $a=0$ or $(0,|a|)$ is a short interval; otherwise, it is called tall. A tuple $a \in M^{n}$ is called short if $|a|:=\left|a_{1}\right|+\cdots+\left|a_{n}\right|$ is short, and tall otherwise. A definable set $X \subseteq M^{n}$ (or its defining formula) is called short if it is in definable bijection with a subset of $(0,1)^{n}$; otherwise, it is called long. Notice that this is compatible, for $n=1$, with the notion of a short interval.

In [Pet1] and [Ed] the authors proved structure theorems about definable sets and functions. (See also [Bel] for an analysis of semi-bounded sets in a different context.) The gist of those theorems was that the definable sets can be decomposed into 'cones', which are bounded sets 'stretched' along some unbounded directions. Conjecture 1 from [Pet3] asks if we can replace 'bounded' by 'short', and 'unbounded' by 'long', in the definition of a cone and still obtain a structure theorem. We answer this affirmatively (the precise terminology to be given in Section 2 below).

Theorem 2.23 (Refined Structure Theorem). Every A-definable set $X \subseteq M^{n}$ is a finite union of A-definable long cones. (In particular, a short set is a 0-long cone.) Furthermore, for every A-definable function $f: X \subseteq R^{n} \rightarrow R$, there is a finite collection $\mathcal{C}$ of $A$-definable long cones, whose union is $X$ and such that $f$ is almost linear with respect to each long cone in $\mathcal{C}$.

As noted in Remark 2.24 below, it is not always possible to achieve disjoint unions in our theorem.

This theorem implies, in particular, a quantifier elimination result down to suitable existential formulas in the spirit of [vdD1] (see Corollary 2.25). The proof of the Refined Structure Theorem involves an induction on the 'long dimension' of definable sets, which is a refinement of the notion of 'linear dimension' from [Ed]. 
We then turn our attention to semi-bounded groups. Groups definable in ominimal structures have been a central object of study in model theory. The climax of that study was the work around Pillay's Conjecture (PC) and Compact Domination Conjecture $(\mathrm{CDC})$, stated in $[\mathrm{Pi} 3]$ and $[\mathrm{HPP}]$, respectively. In the linear case, $(\mathrm{PC})$ was solved in [ElSt] and $(\mathrm{CDC})$ in [El]. The proofs involved a structure theorem for semi-linear groups from [ElSt] that states that every such group is a quotient of a suitable convex subgroup of $\left\langle M^{n},+\right\rangle$ by a lattice. In the field case, (PC) was solved in [HPP] and (CDC) in [HP] (see also [Ot] for an overview of all preceding work). In the case of semi-bounded groups, (PC) was solved in [Pet3] after developing enough theory to allow the combination of the linear and the field cases. The (CDC) for semi-bounded groups remains open. Conjecture 2 from [Pet3] asks if we can prove a structure theorem for semi-bounded groups in the spirit of [ElSt]. In the second part of this paper, we prove a local theorem for semi-bounded groups which we see as a first step towards Conjecture 2 from [Pet3].

The proof of the local theorem involves a new notion of a closure operator in $\mathcal{M}$, the 'short closure operator' scl, which makes $(\mathcal{M}, s c l)$ into a pregeometry. The rising notion of dimension coincides with the long dimension (Corollary 4.10). This allows us to make use of desirable properties of 'long-generic' elements and 'longlarge' sets, by virtue of Claim 4.13 below. The local theorem is the following:

Theorem 5.3 Let $G=\langle G, \oplus\rangle$ be a definable group of long dimension $k$. Then every long-generic element $a$ in $G$ is contained in a $k$-long cone $C \subseteq G$, such that for every $x, y \in C$,

$$
x \ominus a \oplus y=x-a+y .
$$

In particular, on $C, G$ is locally isomorphic to $\left\langle M^{k},+\right\rangle$.

We expect that Theorem 5.3 will be the start point in subsequent work for analyzing semi-bounded groups globally.

Structure of the paper and a few words for the proofs. Section 2 deals with the proof of the Refined Structure Theorem. We first introduce the basic objects of long cones and long dimension, then we generalize several lemmas from [Ed] and finally prove our theorem.

Some difficulties that are incorporated in handling the long dimension are worked out in Section 3, and they are the following: although it is fairly easy to see that a definable set $X$ which is the cartesian product of two definable sets with long dimensions $l$ and $m$ has long dimension $l+m$ (Lemma 2.21(iv)), it is not a priori clear why if a definable set $X$ is the union of a definable family of fibers each of long dimension $m$ over a set of long dimension $n$, then $X$ has long dimension $n+m$. We establish this in Lemma 3.2.

Section 4 deals with the new pregeometry coming from the 'short closure operator'.

In Section 5 we prove the local theorem for semi-bounded groups.

Acknowledgements. I wish to thank M. Edmundo, A. Günaydin and Y. Peterzil for numerous discussions that were helpful in this work. Early steps of this work were carried out during my stay at the Fields Institute in Spring 2009, which I thank for their hospitality. 


\section{SEMI-BOUnded SETS}

2.1. Preliminaries. We assume familiarity with the basic notions from o-minimality, such as the inductive definition of cells either as graphs or 'cylinders' of definable continuous functions, the cell decomposition theorem, dimension, generic elements, definable closure, etc. The reader may consult [vdD2] or [Pi2] for these notions.

Let us fix some notation. For every $i=1, \ldots, n$, we denote by $e_{i}$ the standard $i$-th unit vectors from $\Lambda^{n}$. For $v \in \Lambda$, we denote by $\operatorname{dom}(v)$ the domain of $v$. As observed in [Pet3, Section 6], $\Lambda$ modulo a suitable equivalence relation is an ordered field, implying that for every $\lambda, \mu \in \Lambda, \lambda \mu=\mu \lambda$. Given $a>0$ in $M$, let $M_{a}$ be the subspace of $\langle M,+\rangle$ generated by the interval $(-a, a)$. We let $\Lambda_{a}$ denote the set of restrictions to $M_{a}$ of all those elements in $\Lambda$ whose domain contains $M_{a}$. That is,

$$
\Lambda_{a}=\left\{v_{\uparrow M_{a}}: v \in \Lambda \text { and } M_{a} \subseteq \operatorname{dom}(v)\right\} .
$$

By the above, $\Lambda_{a}$ can easily be equipped with the structure of an ordered field.

A definable function $f: A \subseteq M^{n} \rightarrow M$ is called affine on $A$ if it has form

$$
f\left(x_{1}, \ldots, x_{n}\right)=\lambda_{1} x_{1}+\cdots+\lambda_{n} x_{n}+a,
$$

for some fixed $\lambda_{i} \in \Lambda$ and $a \in M^{n}$. Given a definable function $f: A \times M \rightarrow M$, with $A \subseteq M^{n}$, we denote

$$
\Delta_{t} f(a, x):=f(a, x+t)-f(a, x),
$$

for all $x, t \in M$ and $a \in A$.

Lemma 2.1. Let $f: I \rightarrow M^{n}$ be a definable function, where $I$ is a long interval. If $f(I)$ is short, then $f$ is piecewise constant except for a finite collection of short subintervals of $I$.

Proof. The function $f$ is piecewise strictly monotone or constant. If it were strictly monotone on a long subinterval of $I$, then on that subinterval $f$ would be a definable bijection between a long interval and a short set.

Lemma 2.2. Let $f: X \subseteq M^{n} \rightarrow M$ be a definable function. For every $i=1, \ldots, n$, and $\bar{x}^{i}:=\left(x_{1}, \ldots, x_{i-1}, x_{i+1}, \ldots, x_{n}\right) \in M^{n-1}$, let

$$
X_{\bar{x}^{i}}=\left\{x_{i} \in M:\left(x_{1}, \ldots, x_{n}\right) \in X\right\}
$$

be the fiber of $X$ above $\bar{x}^{i}$ and $f_{\bar{x}^{i}}: X_{\bar{x}^{i}} \rightarrow M$ the map $f_{\bar{x}^{i}}\left(x_{i}\right)=f(\bar{x})$. Consider the set

$$
A=\left\{\bar{a} \in X: \forall i \in\{1, \ldots, n\}, f_{\bar{x}^{i}} \text { is monotone in an interval containing } a_{i}\right\} .
$$

Then $\operatorname{dim}(X \backslash A)<\operatorname{dim}(X)$.

Proof. We may assume that $f$ and $X$ are $\emptyset$-definable. The set $A$ is then also $\emptyset$ definable and it clearly contains every generic element of $X$.

2.2. Long cones. Here we refine the notion of a 'cone' from [Ed]. First let us introduce some notation and terminology. If $v=\left(v_{1}, \ldots, v_{n}\right) \in \Lambda^{n}$ and $t \in M$, we denote $v t:=\left(v_{1} t, \ldots, v_{n} t\right)$. We say that $v_{1}, \ldots, v_{k} \in \Lambda^{n}$ are $M$-independent if for all $t_{1}, \ldots, t_{k} \in M$,

$$
v_{1} t_{1}+\cdots+v_{k} t_{k}=0 \text { implies } t_{1}=\cdots=t_{k}=0 .
$$


If $v=\left(v_{1}, \ldots, v_{n}\right) \in \Lambda^{n}$ and $\mu \in \Lambda$, we denote $\mu v:=\left(\mu v_{1}, \ldots, \mu v_{n}\right)$. We say that $v_{1}, \ldots, v_{k} \in \Lambda^{n}$ are $\Lambda$-independent if for all $\mu_{1}, \ldots, \mu_{k}$ in $\Lambda$,

$$
\mu_{1} v_{1}+\cdots+\mu_{k} v_{k}=0 \text { implies } \mu_{1}=\cdots=\mu_{k}=0 .
$$

Definition 2.3. Let $k \in \mathbb{N}$. A $k$-long cone $C \subseteq M^{n}$ is a definable set of the form

$$
\left\{b+\sum_{i=1}^{k} v_{i} t_{i}: b \in B, t_{i} \in J_{i}\right\}
$$

where $B \subseteq M^{n}$ is a short cell, $v_{1}, \ldots, v_{k} \in \Lambda^{n}$ are $M$-independent and $J_{1}, \ldots, J_{k}$ are long intervals each of the form $\left(0, a_{i}\right), a_{i} \in M^{>0} \cup\{\infty\}$, with $J_{i} \subseteq \operatorname{dom}\left(v_{i}\right)$. So a 0 -long cone is just a short cell. A long cone is a $k$-long cone, for some $k \in \mathbb{N}$. We say that the long cone $C$ is normalized if for each $x \in C$ there are unique $b \in B$ and $t_{1} \in J_{1}, \ldots, t_{k} \in J_{k}$ such that $x=b+\sum_{i=1}^{k} v_{i} t_{i}$. In this case, we write:

$$
C=B+\sum_{i=1}^{k} v_{i} t_{i} \mid J_{i}
$$

In what follows, all long cones are assumed to be normalized, and we thus drop the word 'normalized'. We also often refer to $\bar{v}=\left(v_{1}, \ldots, v_{k}\right) \in M^{n^{2}}$ as the direction of the long cone $C$. If we want to distinguish some $v_{j}$, say $v_{n}$, from the rest of the $v_{i}$ 's, we write:

$$
C=B+\sum_{i=1}^{k-1} v_{i} t_{i}\left|J_{i}+v_{n}\right| J_{n}
$$

Remark 2.4. (i) The $\lambda_{1}, \ldots, \lambda_{n} \in \Lambda^{n}$ are $M$-independent if and only if for any/some $\alpha_{1}, \ldots, \alpha_{n} \in M^{>0}$ the 'open $n$-parallelogram' ([ElSt])

$$
H=\left\{\lambda_{1} t_{1}+\cdots+\lambda_{n} t_{n}:-\alpha_{i}<t_{i}<\alpha_{i}\right\}
$$

has dimension $n$. Indeed, the $(\Rightarrow)$ is straightforward, whereas the $(\Leftarrow)$ is by [El, Corollary 2.5].

(ii) It follows that a (normalized) $k$-long cone $C=B+\sum_{i=1}^{k} v_{i} t_{i} \mid J_{i}$ has dimension $k$ if and only if $B$ is finite. In fact, $\operatorname{dim}(C)=\operatorname{dim}(B)+k$.

Definition 2.5. Let $C=B+\sum_{i=1}^{k} v_{i} t_{i} \mid J_{i}$ be a $k$-long cone and $f: C \rightarrow M$ a definable function. We say that $f$ is almost linear with respect to $C$ if there are $\mu_{1}, \ldots, \mu_{k} \in \Lambda^{n}$ and an extension $\tilde{f}$ of $f$ to $\left\{b+\sum_{i=1}^{k} v_{i} t_{i}: b \in B, t_{i} \in\{0\} \cup J_{i}\right\}$, such that

$$
\forall b \in B, t_{1} \in J_{1}, \ldots, t_{k} \in J_{k}, \tilde{f}\left(b+\sum_{i=1}^{k} v_{i} t_{i} \mid J_{i}\right)=\tilde{f}(b)+\sum_{i=1}^{k} \mu_{i} t_{i} \mid J_{i} .
$$

Remark 2.6. Let $C=B+\sum_{i=1}^{k} v_{i} t_{i} \mid J_{i}$ be a $k$-long cone.

(i) If $f: C \rightarrow M$ is almost linear with respect to $C$, then, since $C$ is normalized, the $\mu_{1}, \ldots, \mu_{k}$ and $\tilde{f}$ as above are unique. For this reason, we often abuse notation and write $f$ for $\tilde{f}$.

(ii) If $B=\{b\}$ and $f: C \rightarrow M$ is a definable function, then $f$ is almost linear with respect to $C$ if and only if $f$ is affine on $C$.

(iii) If $f: C \rightarrow M$ is almost linear with respect to $C$, then $f(C)$ is a $k$-long cone. 
(iv) Let $j \in\{1, \ldots, k\}$ and assume $J_{j}=\left(0, a_{j}\right)$ with $a_{j} \in M$. Then

$$
C=B+v_{j} a_{j}+\sum_{i=1}^{k} v_{i}^{\prime} t_{i} \mid J_{i}
$$

where $v_{j}^{\prime}=-v_{j}$ and for $i \neq j, v_{i}^{\prime}=v_{i}$. If, moreover, $f: C \rightarrow M$ is almost linear with respect to $C$ and of the form

$$
f\left(b+\sum_{i=1}^{k} v_{i} t_{i} \mid J_{i}\right)=f(b)+\sum_{i=1}^{k} \mu_{i} t_{i} \mid J_{i}
$$

then

$$
f\left(b+v_{j} a_{j}+\sum_{i=1}^{k} v_{i}^{\prime} t_{i} \mid J_{i}\right)=f\left(b+v_{j} a_{j}\right)+\sum_{i=1}^{k} \mu_{i}^{\prime} t_{i} \mid J_{i}
$$

where $\mu_{j}^{\prime}=-\mu_{j}$ and for $i \neq j, \mu_{i}^{\prime}=\mu_{i}$.

2.3. Generalizing the Lemma on Subcones [Ed, Lemma 3.4]. In this section we prepare the ground for the proof of the Refined Structure Theorem by suitably generalizing certain lemmas from [Ed].

Lemma 2.7. Let $w, v_{1}, \ldots, v_{m} \in \Lambda^{n}$.

(i) If $w t=v_{1} t_{1}+\cdots+v_{m} t_{m}$ for some $t, t_{1}, \ldots, t_{m} \in M, t \neq 0$, then for every $t^{\prime} \in M$, there are $t_{1}^{\prime}, \ldots, t_{m}^{\prime} \in \operatorname{dom}(w)$ such that $w t^{\prime}=v t_{1}^{\prime}+\cdots+v_{m} t_{m}^{\prime}$.

(ii) If, in addition, $b^{\prime}+w s=b+\sum_{i=1}^{m} v_{i} s_{i}$ for some $b, b^{\prime} \in M^{n}$ and $s, s_{1}, \ldots, s_{m} \in$ $M$, then for every $s^{\prime} \in \operatorname{dom}(w)$, there are $s_{1}^{\prime}, \ldots s_{m}^{\prime} \in M$ such that $b^{\prime}+w s^{\prime}=$ $b+\sum_{i=1}^{m} v_{i} s_{i}^{\prime}$.

Proof. (i) This follows from [ElSt, Lemma 3.4], whose proof used only the fact that $\mathcal{M}$ is an o-minimal expansion of an ordered group. Indeed, if $\left|t^{\prime}\right|<|t|$, then by convexity of the set $A=\left\{v_{1} x_{1}+\cdots+v_{m} x_{m}: x_{i} \in \operatorname{dom}\left(v_{i}\right)\right\}$ and the aforementioned lemma, $w t^{\prime} \in A$. If $\left|t^{\prime}\right|>|t|$, then by convexity of $M^{n} \backslash A$, if it were $w t^{\prime} \notin A$, then $w t \notin A$, a contradiction.

(ii) By (i), there are $p_{1}, \ldots, p_{m} \in M$ such that

$$
b^{\prime}+w s^{\prime}=b^{\prime}+w s+w\left(s^{\prime}-s\right)=b+\sum_{i=1}^{m} v_{i} s_{i}+\sum_{i=1}^{m} v_{i} p_{i}=b+\sum_{i=1}^{m} v_{i}\left(s_{i}-p_{i}\right)
$$

Let $s_{i}^{\prime}=s_{i}-p_{i}$.

Lemma 2.8. Let $w_{1}, \ldots, w_{n} \in \Lambda^{n}$ be $M$-independent and $\lambda_{1}, \ldots, \lambda_{n} \in \Lambda^{n}$. Let $t_{1}, \ldots, t_{n} \in M$ be non-zero elements. Assume that:

$$
\begin{aligned}
w_{1} t_{1} & =\lambda_{1} s_{1}^{1}+\cdots+\lambda_{n} s_{1}^{n} \\
& \vdots \\
w_{n} t_{n} & =\lambda_{1} s_{n}^{1}+\cdots+\lambda_{n} s_{n}^{n}
\end{aligned}
$$


for some $s_{i}^{j} \in M$. Then there non-zero $a_{1}, \ldots, a_{n} \in M$ and $b_{i}^{j} \in M, i, j=1, \ldots, n$, such that:

$$
\begin{aligned}
\lambda_{1} a_{1} & =w_{1} b_{1}^{1}+\cdots+w_{n} b_{1}^{n} \\
& \vdots \\
\lambda_{n} a_{n} & =w_{1} b_{n}^{1}+\cdots+w_{n} b_{n}^{n}
\end{aligned}
$$

Proof. In the Appendix.

Notation. If $J=(0, a)$, we denote $\pm J:=(-a, a)$. Let $C=B+\sum_{i=1}^{m} v_{i} t_{i} \mid J_{i}$ be an $m$-long cone. We set:

$$
\langle C\rangle:=\left\{\sum_{i=1}^{m} v_{i} t_{i}: t_{i} \in \pm J_{i}\right\} .
$$

Lemma 2.9 (Lemma on subcones). If $C^{\prime}=B^{\prime}+\sum_{i=1}^{m^{\prime}} w_{i} t_{i} \mid J_{i}^{\prime}$ and $C=B+$ $\sum_{i=1}^{m} v_{i} t_{i} \mid J_{i}$ are two long cones such that $C^{\prime} \subseteq C \subseteq M^{n}$, then $\left\langle C^{\prime}\right\rangle \subseteq\langle C\rangle$ (and hence $m^{\prime} \leq m$ ).

Proof. Fix $b^{\prime} \in C^{\prime}$ and $j \in\left\{1, \ldots, m^{\prime}\right\}$, and denote for convenience $J:=J_{j}^{\prime}$. Then $\forall u \in J, b^{\prime}+w_{j} u \in C^{\prime} \subseteq C$, so there exist a unique $b \in B$ and, for each $i \in\{1, \ldots, m\}$, a unique $t_{i} \in J_{i}$ such that $b^{\prime}+w_{j} u=b+\sum_{i=1}^{m} v_{i}\left(t_{i}\right)$. This yields the following definable functions:

- $\beta: J \rightarrow B$, with $u \mapsto \beta(u)$

- for each $i \in\{1, \ldots, m\}, t_{i}: J \rightarrow J_{i}$, with $u \mapsto t_{i}(u)$,

where

$$
b^{\prime}+w_{j} u=\beta(u)+\sum_{i=1}^{m} v_{i}\left(t_{i}(u)\right) .
$$

By Lemma 2.1 there is a long subinterval $I \subseteq J$ on which $\beta(u)$ is constant and equal, say, to $b$. Now pick any $u_{1}<u_{2}$ in $I$. We have:

$$
w_{j}\left(u_{2}-u_{1}\right)=\sum_{i=1}^{m} v_{i}\left(t_{i}\left(u_{2}\right)-t_{i}\left(u_{1}\right)\right) .
$$

Hence the condition of Lemma 2.7(i) is satisfied for $w=w_{j}$. The condition of Lemma 2.7 (ii) is also true, since $b^{\prime}+w_{j} u \in C$.

Now fix any $t^{\prime} \in J$. We show that $w_{j} t^{\prime} \in\langle C\rangle$. Let $t_{0} \in J$ be small enough so that $s:=t^{\prime}+t_{0}$ is still in $J$. By Lemma 2.7(ii) and since $b^{\prime}+w_{j} s \in C^{\prime} \subseteq C$, there are $s_{i} \in J_{i}$ such that

$$
b^{\prime}+w_{j} s=b+\sum_{i=1}^{m} v_{i} s_{i} .
$$

Similarly, there are $t_{i}^{0} \in J_{i}$ such that

$$
b^{\prime}+w_{j} t_{0}=b+\sum_{i=1}^{m} v_{i} t_{i}^{0}
$$

By (1) and (2), we conclude that:

$$
w_{j} t^{\prime}=w_{j}\left(s-t_{0}\right)=\sum_{i=1}^{m} v_{i}\left(s_{i}-t_{i}^{0}\right) \in\langle C\rangle .
$$


Finally, the fact that $m^{\prime} \leq m$ follows easily from Lemma 2.8 .

Remark 2.10. Observe that it is not always possible to get $w_{j} t^{\prime} \in\langle C\rangle^{>0}:=$ $\left\{\sum_{i=1}^{m} v_{i} t_{i}: t_{i} \in J_{i}\right\}$, as in the corresponding conclusion of [Ed, Lemma 3.4].

Lemma 2.11. Let $C^{\prime}=B^{\prime}+\sum_{i=1}^{k^{\prime}} v_{i}^{\prime} t_{i}\left|J_{i}^{\prime} \subseteq C=B+\sum_{i=1}^{k} v_{i} t_{i}\right| J_{i}$ be two long cones and $f: C \rightarrow M$ a definable function which is almost linear with respect to $C$. Then $f$ is almost linear with respect to $C^{\prime}$.

Proof. By the Lemma on Subcones, for each $j=1, \ldots, k^{\prime}$, there is $t>0$ such that $v_{j}^{\prime} t \in\langle C\rangle$. It is then an easy exercise to check that $f$ is affine in each $v_{j}^{\prime}$; that is, for every $b^{\prime} \in B^{\prime}, t_{1} \in J_{1}^{\prime}, \ldots, t_{k} \in J_{k}^{\prime}$, and $s>0$ such that $t_{j}+s \in J_{j}^{\prime}$,

$$
f\left(b^{\prime}+\sum_{\substack{i \neq j \\ 0<i, j \leq k^{\prime}}} v_{i}^{\prime} t_{i}+v_{j}^{\prime}\left(t_{j}+s\right)\right)-f\left(b^{\prime}+\sum_{\substack{i \neq j \\ 0<i, j \leq k^{\prime}}} v_{i}^{\prime} t_{i}+v_{j}^{\prime} t_{j}\right)
$$

is linear. This exactly means that $f$ is almost linear with respect to $C^{\prime}$.

2.4. Some more lemmas. The proofs of the following two lemmas are straightforward computations and are left to the reader.

Lemma 2.12. Any $v_{1}, \ldots, v_{l} \in \Lambda^{n}$ are $\Lambda$-independent if and only if they are $M$ independent.

Lemma 2.13. Let $v_{1}, \ldots, v_{l} \in M^{n}$ and denote by $\pi: M^{n} \rightarrow M^{n-1}$ the usual projection. The following are equivalent:

(i) There are $\lambda_{1}, \ldots, \lambda_{n-1} \in \Lambda$, such that for all $t_{1}, \ldots, t_{l} \in M$ with $t_{i} \in \operatorname{dom}\left(v_{i}\right)$, $v_{1} t_{1}+\cdots+v_{l} t_{l}$ has form:

$$
v_{1} t_{1}+\cdots+v_{l} t_{l}=\left(a_{1}, \ldots, a_{n-1}, \lambda_{1} a_{1}+\cdots+\lambda_{n-1} a_{n-1}\right) .
$$

(ii) $\pi\left(v_{1}\right), \ldots, \pi\left(v_{l}\right)$ are $\Lambda$-independent.

Corollary 2.14. If $D=b+\sum_{i=1}^{l} v_{i} t_{i} \mid J_{i} \subseteq M^{n}$ is an l-long cone, then some projection onto $M^{l}$ is a bijection whose image is an l-long cone.

Proof. By Lemmas 2.12 and 2.13.

For the next lemma, recall the the notation $M_{a}$ from the beginning of this section.

Lemma 2.15. Let $a>0$ in $M$ abd $v_{1}, \ldots, v_{l} \in\left(\Lambda_{a}\right)^{n}$ be $M$-independent. Then, for every $t_{1}, \ldots, t_{l} \in M_{a}$,

$$
v_{1} t_{1}+\cdots+v_{l} t_{l} \text { is short } \Rightarrow t_{1}, \ldots, t_{l} \text { are short. }
$$

Proof. Since $v_{i}=\left(\begin{array}{c}v_{i}^{1} \\ \vdots \\ v_{i}^{n}\end{array}\right), i=1, \ldots, l$, are $\Lambda$-independent, the matrix

$$
A=\left(\begin{array}{ccc}
v_{1}^{1} & \ldots & v_{l}^{1} \\
\vdots & \ldots & \vdots \\
v_{1}^{n} & \ldots & v_{l}^{n}
\end{array}\right)
$$


has rank $l$. Let $B$ be an $l \times l$ submatrix of $A$ of $\operatorname{rank} l$. Then $B\left(\begin{array}{c}t_{1} \\ \vdots \\ t_{l}\end{array}\right)=\left(\begin{array}{c}s_{1} \\ \vdots \\ s_{l}\end{array}\right)$, for some short $s_{1}, \ldots, s_{l} \in M$. Hence $\left(\begin{array}{c}t_{1} \\ \vdots \\ t_{l}\end{array}\right)=B^{-1}\left(\begin{array}{c}s_{1} \\ \vdots \\ s_{l}\end{array}\right)$ and each row of the last matrix consists of a short element.

Corollary 2.16. Let $C=b+\sum_{i=1}^{k} v_{i} t_{i} \mid J_{i}$ be a k-long cone. Let $\lambda \in \Lambda^{k}$ be such that for some positive $t \in M, \lambda t \in\langle C\rangle$. Then there is a tall $b \in M$ such that $\lambda b \in\langle C\rangle$.

Proof. Without loss of generality, we may assume that for some $a$, each $v_{i} \in\left(\Lambda_{a}\right)^{n}$. Fix $i$. Let $\alpha=\sup \{x \in M: \lambda x \in\langle C\rangle\}$. It is easy to see that $\alpha=v_{1} t_{1}+\cdots+v_{k} t_{k}$, with at least one of $t_{1}, \ldots, t_{k}$, say $t_{i}$, equal to $\left|J_{i}\right|$. Hence, by Lemma $2.15, \alpha$ is tall. Take $b=\frac{1}{2} \alpha$ (since $\alpha$ is not in $\langle C\rangle$ ).

Corollary 2.17. Let $C \subseteq C^{\prime}$ be two k-long cones and let $\bar{v}$ be the direction of $C^{\prime}$. Then there is a k-long cone of direction $\bar{v}$ contained in $C$.

Proof. By the Lemma on Subcones, Lemma 2.8 and Corollary 2.16.

2.5. Long dimension. Here we suitably refine the notion of linear dimension from [Ed].

Definition 2.18. Let $Z \subseteq M^{n}$ be a definable set. Then the long dimension of $Z$ is defined to be

$$
\lg \operatorname{dim}(Z)=\max \{k: Z \text { contains a } k \text {-long cone }\} .
$$

The following lemma is a kind of converse to the Lemma on Subcones:

Lemma 2.19. Let $C=b+\sum_{i=1}^{k} v_{i} t_{i} \mid J_{i}$ be a k-long cone. Let $w_{1}, \ldots, w_{k} \in \Lambda^{n}$ be $M$-independent such that for every $i$, there is a positive $s_{i} \in M, w_{i} s_{i} \in\langle C\rangle$. Then there is a $k$-long subcone $C^{\prime} \subseteq C$ of the form $C^{\prime}=c+\sum_{i=1}^{k} w_{i} t_{i} \mid\left(0, \kappa_{i}\right)$, for some tall $\kappa_{i} \in M$.

Proof. By Corollary 2.16, we may assume that each $s_{i}$ is tall. Assume $J_{i}=\left(0, a_{i}\right)$. Let $c=b+\sum_{i=1}^{k} \frac{1}{2} v_{i} a_{i}$ and for each $i$, let $\kappa_{i}=\frac{1}{2 k}\left|s_{i}\right|$. Using Lemma 2.7(i), one can easily check that $C^{\prime}=c+\sum_{i=1}^{k} w_{i} t_{i} \mid\left(0, \kappa_{i}\right) \subseteq C$.

The following corollary will not be used until Section 5 .

Corollary 2.20. Let $X \subseteq M^{n}$ be a definable set of long dimension $k$. If $C \subseteq X \times X$ is a $2 k$-long cone, then there are $k$-long cones $C_{1}, C_{2} \subseteq X$, such that $C_{1} \times C_{2} \subseteq C$.

Proof. We may assume that $C=b+\sum_{i=1}^{2 k} v_{i} t_{i} \mid J_{i}$. Let $\pi: M^{2 n} \rightarrow M^{2 k}$ be the bijection given by Corollary 2.14. Denote by $p_{1}, p_{2}: M^{n} \rightarrow M^{k}$ the obvious projections (indeed, bijections) from $M^{n}$ onto $M^{k}$ so that $\pi(C) \subseteq p_{1}(X) \times p_{2}(X)$. By Lemma $2.19, \pi(C)$ contains a $2 k$-long cone

$$
C^{\prime}=\left(b_{1}, b_{2}\right)+\sum_{i=1}^{2 k} e_{i} t_{i} \mid(0, a) .
$$

The pullbacks

$$
C_{1}=p_{1}^{-1}\left(b_{1}+\sum_{i=1}^{k} e_{i} t_{i} \mid(0, a)\right) \cap X
$$


and

$$
C_{2}=p_{2}^{-1}\left(b_{2}+\sum_{i=1}^{k} e_{i} t_{i} \mid(0, a)\right) \cap X
$$

have the desired properties.

Lemma 2.21. Let $X, Y, X_{1}, \ldots, X_{k}$ be definable sets. Then:

(i) $\lg \operatorname{dim}(X) \leq \operatorname{dim}(X)$.

(ii) $X \subseteq Y \subseteq M^{n} \Rightarrow \operatorname{lgdim}(X) \leq \operatorname{lgdim}(Y) \leq n$.

(iii) If $C$ is a $n$-long cone, then $\operatorname{lgdim}(C)=n$.

(iv) $\lg \operatorname{dim}(X \times Y)=\lg \operatorname{dim}(X)+\lg \operatorname{dim}(Y)$.

(v) $\lg \operatorname{dim}\left(X_{1} \cup \cdots \cup X_{k}\right)=\max \left\{\operatorname{lgdim}\left(X_{1}\right), \ldots, \lg \operatorname{dim}\left(X_{k}\right)\right\}$.

Proof. (i) and (ii) are clear, by Remark 2.4. Item (iii) follows from the Lemma on Subcones 2.9. The proof of (iv) is word-by-word the same with the proof of [EdEl, Fact 2.2(3)] after replacing 'ldim' by 'Igdim' and the notion of a cone by that of a long cone we have here.

For (v), we prove by parallel induction on $n \geq 1$ the following two statements.

$(1)_{n}$ For all $k$ and all definable $X_{1}, \ldots, X_{k}$ such that $\operatorname{lgdim}\left(X_{1} \cup \cdots \cup X_{k}\right)=n$, there exists $i \in\{1, \ldots, k\}$ such that $\lg \operatorname{dim}\left(X_{i}\right)=n$.

$(2)_{n}$ Let $C \subseteq M^{n}$ be an n-long cone. For any definable set $X \subseteq C$ with $\operatorname{dim}(X) \leq$ $n-1$ we have $\lg \operatorname{dim}(C \backslash X)=n$.

STEP I: $(2)_{1}$ follows from [Pet3, Lemma 3.4(2)].

STEP II: $(1)_{n-1}$ and $(2)_{l}$ for $l \leq n-1$ imply $(2)_{n}$, for $n \geq 2$. Assume $(1)_{n-1}$ and $(2)_{l}$ for all $l \leq n-1$. We perform a sub-induction on $\operatorname{dim}(X)$. Observe that after some suitable linear transformation we may assume that $C$ has form

$$
C=\sum_{i=1}^{n} e_{i} t_{i} \mid J_{i}
$$

where the $e_{i}$ 's are the standard basis vectors.

If $\operatorname{dim}(X)=0$, then $X$ is finite and, without loss of generality, we may assume that $X$ contains only one point $a$. Then it is easy to see that $C \backslash\{a\}$ contains $2^{n}$ disjoint long cones of the form $a+\sum_{i=1}^{n} e_{i} t_{i} \mid J_{i}^{\prime}$ such that, for at least one of them, all $J_{i}^{\prime \prime}$ 's are long.

Suppose the result holds for all $X$ with $\operatorname{dim}(X) \leq l<n-1$, and assume now that $\operatorname{dim}(X)=l+1$. If $l+1<n-1$, then $\operatorname{dim}(\pi(X)) \leq n-2$ and by $(2)_{n-1}$, $\lg \operatorname{dim}(\pi(C) \backslash \pi(X))=n-1$, which implies that $\lg \operatorname{dim}(C \backslash X)=n$, by (iv).

So now assume that $\operatorname{dim}(X)=n-1$. By cell decomposition and by the SubInductive Hypothesis, we may assume that $X$ is a finite union of cells $X_{1}, \ldots, X_{k}$, each of dimension $n-1$. We perform a second sub-induction on $k$.

Base Step: suppose $k=1$. If $X_{1}$ is not the graph of a function or $\lg \operatorname{dim}\left(X_{1}\right)<n-1$, then by $(2)_{n-1}$ or $(1)_{n-1}$, respectively, we have $\operatorname{lgdim}\left(\pi(C) \backslash \pi\left(X_{1}\right)\right)=n-1$, which implies $\lg \operatorname{dim}(C \backslash X)=n$, by (iv). Thus it remains to examine the case where $X_{1}$ is the graph of a function $f: \pi\left(X_{1}\right) \rightarrow M$ and $\operatorname{lgdim}\left(X_{1}\right)=n-1$. In this case, $\lg \operatorname{dim}\left(\pi\left(X_{1}\right)\right)=\lg \operatorname{dim}\left(X_{1}\right)=n-1$, where the first equality is by Lemma 2.13 . Let $D \subseteq \pi\left(X_{1}\right)$ be a $(n-1)$-long cone. Let

$$
A=\left\{\bar{a} \in D: \forall i \in\{1, \ldots, n-1\}, f_{\bar{x}^{i}} \text { is monotone around } a_{i}\right\} .
$$


according to the notation of Lemma 2.2. By that lemma,

$$
\operatorname{dim}(D \backslash A)<\operatorname{dim}(D)=n-1 .
$$

Hence, by $(2)_{n-1}, A$ contains an $(n-1)$-long cone $E$, and by Lemma 2.19 , we may assume that $E=b+\sum_{i=1}^{n-1} e_{i} t_{i} \mid(0, \kappa)$, for some tall $\kappa$. Let $\bar{a}=b+\sum_{i=1}^{n-1} e_{i} \frac{1}{2} \kappa$. Since $f$ is continuous on $E$, each $f_{\bar{x}^{i}}$ is monotone on its domain $(0, \kappa)$. Without loss of generality, we may assume that $\forall i \in\{1, \ldots, n-1\}, f_{\bar{x}^{i}}$ is increasing on $(0, \kappa)$. We split into two cases:

Case 1: $f(\bar{a})$ is short. Then the $n$-long cone

$$
E_{1}=(b, f(\bar{a}))+\sum_{i=1}^{n-1} e_{i} t_{i}\left|(0, \kappa / 2)+e_{n} t_{n}\right| J_{n} / 2
$$

is contained in $X_{1}$.

Case 2: $f(\bar{a})$ is tall. Then the $n$-long cone

$$
E_{1}=(\bar{a}, 0)+\sum_{i=1}^{n-1} e_{i} t_{i}\left|(0, \kappa / 2)+e_{n} t_{n}\right| J_{n} / 2
$$

is contained in $X_{1}$. This completes the case $k=1$.

Inductive Step: suppose the result holds for any $X$ which is a union of less than $k$ cells of dimension $n-1$, and assume now that $X$ is the union of the cells $X_{1}, \ldots, X_{k}$, each of dimension $n-1$. By Second Sub-Inductive Hypothesis, there is an $n$-long cone $F$ contained in $C \backslash\left(X_{1} \cup \cdots \cup X_{k-1}\right)$. Now, we reduce to the Base Step for $C$ equal to $F$ and $X_{1}$ equal to $X_{k}$. This completes the proof of the second sub-induction, as well as that of Step II of the original induction.

STEP III: $(2)_{n} \Rightarrow(1)_{n}$. Without loss of generality, we may assume that $k=2$ and $X_{1}$ and $X_{2}$ are disjoint. Since $\lg \operatorname{dim}\left(X_{1} \cup X_{2}\right)=n$, we may also assume that $X_{1} \cup X_{2}$ is an $n$-long cone $C$ of dimension $n$. If $X=b d\left(X_{1}\right) \cup b d\left(X_{2}\right)$, then $\operatorname{dim}(X) \leq n-1$. By $(2)_{n}$, we conclude that either $X_{1}$ or $X_{2}$ contains an $n$-long cone.

Lemma 2.22. Let $X=(f, g)_{\pi(X)}$ be a cylinder in $M^{n+1}$ such that $\pi(X)$ is a $k$-long cone and $f$ and $g$ are almost linear with respect to $\pi(X)$. If there is an $x \in \pi(X)$ such that $\pi^{-1}(x)$ is long, then $\lg \operatorname{dim}(X)=k+1$.

Proof. If $k=0$, then there is an 1-long cone $\pi^{-1}(x) \subseteq X$. Now assume $k>0$ and that for some $x \in \pi(X), \pi^{-1}(x)=(f(x), g(x))$ is long. Since $f, g$ are almost linear on $\pi(X)$, there is clearly a $k$-long cone $C_{x}=x+\sum_{i=1}^{k} v_{i} t_{i} \mid\left(0, a_{i}\right) \subseteq \pi(X)$ such that for each element $y \in C_{x}, g(y)-f(y)$ must be tall. Let $\alpha=\inf \left\{g(y)-f(y): y \in C_{x}\right\}$. Since $f$ is affine,

$$
\forall t_{1} \in J_{1}, \ldots, t_{k} \in J_{k}, f\left(x+\sum_{i=1}^{k} v_{i} t_{i} \mid J_{i}\right)=f(x)+\sum_{i=1}^{k} \mu_{i} t_{i} \mid J_{i},
$$

for some $\mu_{1}, \ldots, \mu_{k} \in \Lambda^{n}$. Then clearly the $(k+1)$-long cone

$$
(x, f(x))+\sum_{i=1}^{k}\left(v_{i}, \mu_{i}\right) t_{i}\left|J_{i}+e_{n+1} t_{k+1}\right|(0, \alpha)
$$

is contained in $X$. 
2.6. Proof of the Refined Structure Theorem. We are now in a position to prove the main result of this paper.

Theorem 2.23. (Refined Structure Theorem). Let $X \subseteq M^{n}$ be an A-definable set. Then

(i) $X$ is a finite union of $A$-definable long cones.

(ii) If $X$ is the graph of an $A$-definable function $f: Y \rightarrow M$, for some $Y \subseteq$ $M^{n-1}$, then there is a finite collection $\mathcal{C}$ of A-definable long cones, whose union is $Y$ and such that $f$ is almost linear with respect to each long cone in $\mathcal{C}$.

Proof. By cell decomposition we may assume that $X$ is an $A$-definable cell. We prove (i) and (ii), along with (iii) below, by induction on $\langle n, \lg \operatorname{dim}(X)\rangle$.

(iii) In the notation from (ii), $Y$ contains an A-definable $\operatorname{lgdim}(Y)$-long cone such that $f$ is almost linear with respect to it.

If $n=1$, then (i), (ii) and (iii) are clear. Assume the Inductive Hypothesis (IH): (i), (ii) and (iii) hold for $\{\langle n, k\rangle\}_{k \leq n}$, and let $X \subseteq M^{n+1}$ with $\operatorname{lgdim}(X)=k \leq n+1$.

Case (I): $\operatorname{dim}(X)<n+1$. So, after perhaps permuting the coordinates, we may assume that $X$ is the graph of a continuous $A$-definable function $f: Y \rightarrow M$.

(i) This is clear, by (IH)(ii) and Remark 2.6(iii).

(ii) By (IH)(i), we may further assume that $Y=B^{\prime}+\sum_{i=1}^{k} v_{i} t_{i} \mid J_{i}$ is an $A$ definable $k$-long cone, where $k \leq n$. By applying a suitable linear transformation, we may assume that $Y=B+\sum_{i=1}^{k} e_{n-k+i} t_{i} \mid J_{i}$. Let $\pi: M^{n} \rightarrow M^{n-1}$ be the usual projection. By [Pet3, Lemma 4.10] and its proof, there are $A$-definable linear functions $\lambda_{1}, \ldots, \lambda_{l}, A$-definable functions $a_{0}, \ldots, a_{m}: \pi(Y) \rightarrow M$ and a short positive element $b \in \operatorname{dcl}(A)$ of $M$, such that for every $x \in \pi(Y)$,

- $0=a_{0}(x) \leq a_{1}(x) \leq \cdots \leq a_{m-1}(x) \leq a_{m}(x)=e_{n}\left|J_{k}\right|$

- for every $i$, either $\left|a_{i+1}(x)-a_{i}(x)\right|<b$ or the map $t \mapsto \Delta_{t} f\left(\bar{x}, a_{i}(x)\right)$ on $\left(0, a_{i+1}(x)-a_{i}(x)\right)$ is the restriction of some $\lambda_{j}$; that is

$$
f\left(\bar{x}, a_{i}(x)+t\right)-f\left(\bar{x}, a_{i}(x)\right)=\lambda_{j}(t) .
$$

For every $z=(x, y) \in Y$, let $b_{z}:=a_{i+1}(x)-a_{i}(x)$, where $y \in\left(a_{i}(x), a_{i+1}(x)\right)$. Observe that $b_{z} \in d c l(\emptyset)$. Set

$$
Y_{0}=\left\{z \in Y: b_{z} \geq b\right\},
$$

and consider (by cell decomposition) a partition $\mathcal{C}$ of $Y_{0}$ into cells so that for every $Z \in \mathcal{C}$

- there is some $\lambda_{j}$ such that the restriction of $f$ on $Z$ satisfies (3) above,

- $Z$ is contained in $\left\{(x, y): a_{i}(x) \leq y \leq a_{i+1}(x)\right\}$.

By (IH)(ii), there is a finite collection $\mathcal{C}^{\prime}$ of $A$-definable long cones, whose union is $\pi(Z)$ and such that each $a_{i}$ is almost linear with respect to each $C \in \mathcal{C}^{\prime}$. By (IH)(i), there is a finite collection $\mathcal{C}^{\prime \prime}$ of $A$-definable long cones, whose union is $Z \cap \pi^{-1}(C)$. Observe now that $Z \cap \pi^{-1}(C)$ is contained in some long cone $W$ on which $f$ is almost linear; namely, if $C=D+\sum_{i=1}^{l} w_{i} t_{i} \mid K_{i}$, then $W$ is of the form

$$
W=D \times\{d\}+\sum_{i=1}^{l} w_{i} t_{i}\left|K_{i}+e_{n} t_{n}\right| K_{n},
$$


where $K_{n}$ is a long interval of length equal to $\max \left\{a_{i+1}(x)-a_{i}(x): x \in C\right\}$. By Lemma 2.11, we conclude that $f$ is almost linear with respect to each long cone in $\mathcal{C}^{\prime \prime}$

It remains to prove (i) for $Y \backslash Y_{0}$. But this is given by (IH)(ii), since, in fact, $\operatorname{lgdim}\left(Y \backslash Y_{0}\right)<k$ : assuming not, apply (IH)(iii) to get a $k$-long cone $C \subseteq Y \backslash Y_{0} \subseteq$ $Y$. By Corollary 2.17, there is a tall $a \in M$ such that $e_{n} a \in C$. But then $f$ is linear in $x_{n}$ on some long interval contained in $Y \backslash Y_{0}$, a contradiction. Hence $\lg \operatorname{dim}\left(Y \backslash Y_{0}\right)<k$.

(iii) In the above notation, for every $i \in\{0, \ldots, m-1\}$, the set

$$
P_{i}:=\left\{\bar{x} \in \pi(Y): a_{i+1}(\bar{x})-a_{i}(\bar{x}) \geq b\right\}
$$

is $A$-definable and, since $J_{n}$ is long, $\pi(Y)=\bigcup_{i=0}^{m-1} P_{i}$. By Lemma 2.21(v), one of the $P_{i}$ 's, say $P_{j}$, must have long dimension $k-1$. By (IH)(iii), there is a finite collection $\mathcal{C}^{\prime}$ of $A$-definable long cones, whose union is $W_{j}$ and such that each $a_{j}$ and $a_{j+1}$ are almost linear with respect to each $C \in \mathcal{C}^{\prime}$. By Lemma 2.22, there is an $A$-definable $k$-long cone $E \subseteq Y$ and, as before, $f$ is almost linear with respect to $E$.

Case (II): $\operatorname{dim}(X)=n+1$. The argument in this case is a combination of the proofs of [ElSt, Lemma 3.6] and of [Pet1, Theorem 3.1]. So $X=(g, h)_{Y}$ is a cylinder. By (IH)(ii) and Lemma 2.11, we may assume that $Y=B+\sum_{i=1}^{k} v_{i} t_{i} \mid J_{i}$ is a long cone and that $g, h$ are almost linear with respect to it. Assume they are of the form:

$g\left(b+\sum_{i=1}^{k} v_{i} t_{i} \mid J_{i}\right)=g(b)+\sum_{i=1}^{k} n_{i} t_{i} \mid J_{i}$ and $h\left(b+\sum_{i=1}^{k} v_{i} t_{i} \mid J_{i}\right)=h(b)+\sum_{i=1}^{k} m_{i} t_{i} \mid J_{i}$.

Since $g<h$ on $Y$, it follows that for every $b \in B, g(b)<h(b)$. One of the following two cases must occur:

Case $\left(\mathrm{II}_{a}\right)$ : for all $i=1, \ldots, k$, we have $n_{i}=m_{i}$.

Case $\left(\mathrm{II}_{b}\right)$ : for all $i=1, \ldots, k$, we have $n_{i} \leq m_{i}$, and for at least one $i$ we have $n_{i}<m_{i}$. (We may assume so by Remark 2.6(iv).)

Proof of Case $\left(I I_{a}\right)$. We have

$$
X=\left\{(b, y)+\sum_{i=1}^{k}\left(v_{i}, n_{i}\right) t_{i}: g(b)<y<h(b), b \in B, t_{i} \in J_{i}\right\} .
$$

It is easy to check that, if $(g(b), h(b))$ is a long interval, then

$$
X=\{(b, g(b)): b \in B\}+\sum_{i=1}^{k}\left(v_{i}, n_{i}\right) t_{i}\left|J_{i}+e_{n+1} t_{n+1}\right|(0, h(b)-g(b))
$$

is a $(k+1)$-long cone, and if $(g(b), h(b))$ is short, then

$$
X=\{\{b\} \times(g(b), h(b)): b \in B\}+\sum_{i=1}^{k}\left(v_{i}, n_{i}\right) t_{i} \mid J_{i}
$$

is a $k$-long cone. 
Proof of Case $\left(I I_{b}\right)$. We have

$$
X=\left\{\left(b+\sum_{i=1}^{k} v_{i} t_{i}, y\right): g(b)+\sum_{i=1}^{k} n_{i} t_{i}<y<h(b)+\sum_{i=1}^{k} m_{i} t_{i}, b \in B, t_{i} \in J_{i}\right\} .
$$

Notice that if $h=+\infty$ on $X$ (similarly, if $g=-\infty$ ), then we are done because

$$
X=\{(b, g(b)): b \in B\}+\sum_{i=1}^{k} v_{i} t_{i}\left|J_{i}+e_{n} t_{n}\right|(0,+\infty)
$$

We partition $X$ in the following way, going from "top" to "bottom":

$$
\begin{aligned}
& X_{1}=\left\{\left(b+\sum_{i=1}^{k} v_{i} t_{i}, d\right): h(b)+\sum_{i=1}^{k} n_{i} t_{i}<y<h(b)+\sum_{i=1}^{k} m_{i} t_{i}, b \in B, t_{i} \in J_{i}\right\}, \\
& X_{2}=\left\{\left(b+\sum_{i=1}^{k} v_{i} t_{i}, y\right): y=h(b)+\sum_{i=1}^{k} n_{i} t_{i}, b \in B, t_{i} \in J_{i}\right\}, \\
& X_{3}=\left\{\left(b+\sum_{i=1}^{k} v_{i} t_{i}, y\right): g(b)+\sum_{i=1}^{k} n_{i} t_{i}<y<h(b)+\sum_{i=1}^{k} n_{i} t_{i}, b \in B, t_{i} \in J_{i}\right\} .
\end{aligned}
$$

By Remark 2.6(iii), $X_{2}$ is a $k$-long cone, whereas $X_{3}$ clearly satisfies the condition of Case $\left(\mathrm{II}_{a}\right)$. Hence we only need to account for $X_{1}$.

Let $S_{X_{1}}=\left\{i=1, \ldots, k: n_{i}<m_{i}\right\}$. By induction on $\left|S_{X_{1}}\right|$ we may assume that $\left|S_{X_{1}}\right|=1$. Indeed, if, say, $n_{1}<m_{1}$ and $n_{2}<m_{2}$, then we can partition $X_{1}$ in the following way, going again from "top" to "bottom":

$$
\begin{aligned}
& X_{1}^{\prime}=\left\{\left(b+\sum_{i=1}^{k} v_{i} t_{i}, y\right): h(b)+n_{1} t_{1}+\sum_{i=2}^{k} m_{i} t_{i}<y<h(b)+\sum_{i=1}^{k} m_{i} t_{i}, b \in B, t_{i} \in J_{i}\right\}, \\
& X_{1}^{\prime \prime}=\left\{\left(b+\sum_{i=1}^{k} v_{i} t_{i}, y\right): y=h(b)+n_{1} t_{1}+\sum_{i=2}^{k} m_{i} t_{i}, b \in B, t_{i} \in J_{i}\right\}, \\
& X_{1}^{\prime \prime \prime}=\left\{\left(b+\sum_{i=1}^{k} v_{i} t_{i}, y\right): h(b)+\sum_{i=1}^{k} n_{i} t_{i}<y<h(b)+n_{1} t_{1}+\sum_{i=2}^{k} m_{i} t_{i}, b \in B, t_{i} \in J_{i}\right\} .
\end{aligned}
$$

Observe then that $X_{1}^{\prime \prime}$ is a $k$-long cone, and for $X_{1}^{\prime}$ and $X_{1}^{\prime \prime \prime}$, each of the correspond$\operatorname{ing} S_{X_{1}^{\prime}}$ and $S_{X_{1}^{\prime \prime}}$ has size less than $\left|S_{X_{1}}\right|$.

So assume now that $\left|S_{X_{1}}\right|=1$ with, say, $n_{1}<m_{1}$ and $n_{i}=m_{i}$ for $i>1$. We show that $\left\langle X_{1}\right\rangle$ is the union of long cones which clearly implies that so is $X_{1}$. If $J_{1}=(0, \infty)$, then

$$
\left\langle X_{1}\right\rangle=\left(v_{1}, n_{1}\right) t_{1}\left|J_{1}+\sum_{i=1}^{k}\left(v_{i}, m_{i}\right) t_{i}\right| J_{i}
$$


is already a $(k+1)$-long cone. If $J_{1}=\left(0, a_{1}\right)$, with $a_{1} \in M$, then $\left\langle X_{1}\right\rangle$ is the union of the following $(k+1)$-long cones:

$$
\begin{aligned}
& Y_{1}=\left(v_{1}, n_{1}\right) t_{1}\left|\left(0, \frac{a_{1}}{2}\right)+\left(v_{1}, m_{1}\right) t_{1}\right|\left(0, \frac{a_{1}}{2}\right)+\sum_{i=2}^{k}\left(v_{i}, m_{i}\right) t_{i} \mid J_{i}, \\
& Y_{2}=\left(v_{1}, n_{1}\right) \frac{a_{1}}{2}+\left(v_{1}, n_{1}\right) t_{1}\left|\left(0, \frac{a_{1}}{2}\right)+\sum_{i=2}^{k}\left(v_{i}, m_{i}\right) t_{i}\right| J_{i}+e_{n} t_{n} \mid\left(0, \frac{\left(m_{1}-n_{1}\right) a_{1}}{2}\right) \\
& Y_{3}=\left(v_{1}, n_{1}\right) \frac{a_{1}}{2}+\left(v_{1}, m_{1}\right) t_{1}\left|\left(0, \frac{a_{1}}{2}\right)+\sum_{i=2}^{k}\left(v_{i}, m_{i}\right) t_{i}\right| J_{i}+e_{n} t_{n} \mid\left(0, \frac{\left(m_{1}-n_{1}\right) a_{1}}{2}\right)
\end{aligned}
$$

Remark 2.24. As opposed to the corresponding results from [Ed] and [Pet1], it is not always possible to achieve a disjoint union in (i) or (ii). We leave it to the reader to verify that the following set cannot be written as a disjoint union of long cones: let $X$ be the 'triangle' with corners the origin, the point $(a, a)$ and the point $(0,2 a)$, for some long element $a$.

As a first corollary, we obtain a quantifier elimination result down to suitable existential formulas in the spirit of [vdD1].

Corollary 2.25. Every definable subset $X \subseteq M^{m}$ is a boolean combination of subsets of $M^{m}$ defined by

$$
\exists y_{1} \ldots \exists y_{m} B\left(y_{1}, \ldots, y_{m}\right) \wedge \varphi\left(x_{1}, \ldots, x_{m}, y_{1}, \ldots, y_{m}\right),
$$

where $B(y)$ is a short formula and $\varphi(x, y)$ is a quantifier-free $\mathcal{L}_{\text {lin }}$-formula. In fact, $X$ is a finite union of such sets.

Another corollary is the following

Corollary 2.26. If $f: X \rightarrow M^{n}$ is a definable injective function, then $\lg \operatorname{dim}(X)=$ $\lg \operatorname{dim}(f(X))$.

Proof. By the Refined Structure Theorem and Remark 2.6(iii).

\section{ON DEFINABILITY OF LONG DIMENSION}

The following example shows that we lack 'definability of long dimension'.

Example 3.1. Let $a>0$ be a tall element and let

$$
X=\{(x, y): 0 \leq x \leq a, 0 \leq y \leq x\} .
$$

Denote by $\pi: M^{2} \rightarrow M$ the usual projection. Then, by [Pet3, Proposition 3.6], the set

$$
X_{1}=\left\{x \in[0, a]: \pi^{-1}(x) \text { has long dimension } 1\right\}
$$

is not definable.

However, $X_{1}$ clearly contains a 'suitable' definable set; namely, a definable set of long dimension 1. It follows from the lemmas of this section that the set of fibers of any definable set $X$ of a given long dimension $l$ always lies between two definable sets each of long dimension $\lg \operatorname{dim}(X)-l$ (Corollary 3.4 below). 
Lemma 3.2. Let $X \subseteq M^{n+m}$ be a definable set such that the projection $\pi(X)$ onto the first $n$ coordinates has long dimension $k$. Let $0 \leq l \leq m$. Then

(i) $\operatorname{lgdim}(X) \leq k+m$.

(ii) $\lg \operatorname{dim}(X) \geq k+l$ if and only if $\pi(X)$ contains a $k$-long cone $C$ such that every fiber $X_{c}, c \in C$, has long dimension $\geq l$.

Proof. (i) By Lemma 2.21(ii)\&(iv), since $X \subseteq \pi(X) \times M^{m}$.

(ii) $(\Leftarrow)$ Assume that every fiber $X_{c}, c \in C$, has long dimension $l$. We prove that $\lg \operatorname{dim}(X) \geq k+l$ by induction on $k$. For $k=0$, it is clear, since any fiber above $C$ contains a $l$-long cone. Now assume that it is proved for $\lg \operatorname{dim}(C)<k$, and let $\lg \operatorname{dim}(C)=k$. Clearly, we may assume that $\pi(X)=C$. For the sake of contradiction, assume $\lg \operatorname{dim}(X)<k+l$. By the Refined Structure Theorem, $X$ can be covered by finitely many long cones $X_{1}, \ldots, X_{s}$, each with $\lg \operatorname{dim}\left(X_{i}\right)<k+l$. By the inductive hypothesis, each $\pi\left(X_{i}\right)$ has long dimension $<k$. But then $C=$ $\pi\left(X_{1}\right) \cup \cdots \cup \pi\left(X_{s}\right)$ must have long dimension $<k$, a contradiction.

$(\Rightarrow)$ This is clearly equivalent to the following:

Claim. Let

$$
X_{l}=\left\{x \in \pi(X): \pi^{-1}(x) \text { has long dimension } \geq l\right\}
$$

Then there is a definable set $Y_{l} \subseteq X_{l}$, such that

$$
\lg \operatorname{dim}\left(Y_{l}\right)=\lg \operatorname{dim}(X)-l .
$$

The proof of the Claim is by induction on $m$.

Base Step: $m=l=1$. By cell decomposition, $X$ is a finite union of cells, and by the Refined Structure Theorem the domain of each cell is a finite union of long cones such that the corresponding restrictions of the defining functions of the cell are almost linear with respect to each of the long cones. If a cell is a graph of a function, or if its domain has long dimension $<k$, then clearly its long dimension is at most $k$. Hence $X$ contains a cylinder $X_{1}=(f, g)_{\pi\left(X_{1}\right)}$, where $\pi\left(X_{1}\right)$ is a $k$-long cone, such that $X_{1}$ contains a $(k+1)$-long cone $C=b+\sum_{i=1}^{k+1} v_{i} t_{i} \mid\left(0, \alpha_{i}\right)$. We will first show that for some elements $x, y \in \bar{C}$ in the closure of $C$, with $\forall i=1, \ldots, n$, $x_{i}=y_{i}$, and $(y-x)_{n+1}$ tall. This is straightforward and we only sketch its proof.

The projection $\pi(C)$ is a union of long cones whose directions are tuples with elements from the set $\left\{v_{1}, \ldots, v_{k+1}\right\}$. By (i) and Lemma $2.21(\mathrm{v})$, there must be subset of $\left\{v_{1}, \ldots, v_{k+1}\right\}$ of $k$ elements, say $\left\{v_{1}, \ldots, v_{k}\right\}$, whose projections onto the first $n$-coordinates is an $M$-independent set. Without loss of generality, assume $A=\left\{v_{1}, \ldots, v_{k}\right\}$. It is then an easy exercise to see that there is an element $y=$ $v_{1} t_{1}+\cdots+v_{k+1} t_{k+1} \in \bar{C}$, such that the element

$$
x=\min \left\{z \in \bar{C}: \forall i \leq n, z_{i}=y_{i}\right\}
$$

has form $x=v_{1} s_{1}+\cdots+v_{k+1} s_{k+1} \in \bar{C}$ such that $t_{k+1}-s_{k+1}$ is long. But then $y-x$ must be tall, by Lemma 2.15. It follows that $(y-x)_{n+1}$ must be tall.

Now, we conclude that there is $x \in \pi\left(X_{1}\right)$, such that $\pi^{-1}(x)=(f(x), g(x))$ is long. Since $f, g$ are almost linear on $\pi\left(X_{1}\right)$, it is easy to see that there is a $k$ long cone $C_{x}=x+\sum_{i=1}^{k} v_{i} t_{i} \mid\left(0, a_{i}\right) \subseteq \pi\left(X_{1}\right)$ such that for each element $y \in C_{x}$, $g(y)-f(y)$ is tall. We let $Y_{l}=C_{x}$. Since, by (i), $k \geq \operatorname{lgdim}(X)-1$, we are done. 
Inductive Step: assume we know the lemma for every $n$ and $X \subseteq M^{n} \times M^{m}$, and let $X \subseteq M^{n} \times M^{m+1}$. Let $q: M^{n+m+1} \rightarrow M^{n+m}$ and $r: M^{n} \times M^{m} \rightarrow M^{n}$ be the usual projections. Of course, $\pi=r \circ q$.

Case (I). $\lg \operatorname{dim}(q(X))=\operatorname{lgdim}(X)$. In this case, by the Inductive Hypothesis, the set

$$
q(X)_{l}=\left\{x \in \pi(X): \lg \operatorname{dim}\left(r^{-1}(x)\right) \geq l\right\}
$$

contains a definable set $A$ such that

$$
\lg \operatorname{dim}(A)=\lg \operatorname{dim}(q(X))-l=\lg \operatorname{dim}(X)-l .
$$

Since, clearly, $q(X)_{l} \subseteq X_{l}$, we are done.

Case (II). $\lg \operatorname{dim}(q(X))=\lg \operatorname{dim}(X)-1$. Let

$$
Y_{1}=\left\{x \in q(X): \lg \operatorname{dim}\left(q^{-1}(x)\right)=1\right\} .
$$

By the Base Step, $Y_{1}$ contains some definable set $Y$ with $\lg \operatorname{dim}(Y)=\lg \operatorname{dim}(X)-1$. By the Inductive Hypothesis, the set

$$
Y_{l}=\left\{x \in r(Y): \lg \operatorname{dim}\left(r^{-1}(x)\right) \geq l-1\right\}
$$

contains a definable set $A$ with

$$
\lg \operatorname{dim}(A)=\lg \operatorname{dim}(Y)-(l-1)=\lg \operatorname{dim}(X)-l .
$$

But clearly $X_{l}$ contains $A$ and hence we are done.

On the other hand, we have the following lemma. It will not be essential until the proof of Proposition 5.4.

Lemma 3.3. Let $X \subseteq M^{n+m}$ be a definable set and denote by $\pi: M^{n+m} \rightarrow M^{n}$ the usual projection. For $0 \leq l \leq m$, let

$$
X_{l}=\left\{x \in \pi(X): \pi^{-1}(x) \text { has long dimension } \geq l\right\} .
$$

Then there is a definable subset $Z_{l} \subseteq \pi(X)$ with $X_{l} \subseteq Z_{l}$ such that

$$
\lg \operatorname{dim}\left(Z_{l}\right)=\lg \operatorname{dim}(X)-l .
$$

Proof. The proof is by induction on $m$. For any $m$, if $l=0$, then take $Z_{l}=\pi(X)$, since, by Lemma $3.2($ ii) $) \lg \operatorname{dim}(\pi(X)) \leq \lg \operatorname{dim}(X)$.

Base Step: $m=1$. Let $X \subseteq M^{n} \times M$ and $l=1$. By cell decomposition and Lemma 2.21(v), we may assume that $X$ is a cell. If $X$ is the graph of a function, then let $Z_{l}$ be any subset of $\pi(X)$ of long dimension $\lg \operatorname{dim}(X)-1$. So assume $X$ is the cylinder $(f, g)_{\pi(X)}$ between two continuous functions $f$ and $g$. By the Refined Structure Theorem, we may further assume that $\pi(X)$ is a long cone such that $f$ and $g$ are both almost linear with respect to it. If $\lg \operatorname{dim}(\pi(X))=\lg \operatorname{dim}(X)-1$, then take $Z_{l}=\pi(X)$. If $\lg \operatorname{dim}(\pi(X))=\lg \operatorname{dim}(X)$, then by Lemma 2.22, for every $x \in \pi(X), \pi^{-1}(x)$ is short, in which case we let again $Z_{l}$ be any subset of $\pi(X)$ of long dimension $\lg \operatorname{dim}(X)-1$.

Inductive Step: assume we know the lemma for every $n$ and $X \subseteq M^{n} \times M^{m}$, and let $X \subseteq M^{n} \times M^{m+1}$. Let $q: M^{n+m+1} \rightarrow M^{n+m}$ and $r: M^{n} \times M^{m} \rightarrow M^{n}$ be the usual projections. Let

$$
Y_{1}=\left\{x \in q(X): \lg \operatorname{dim}\left(q^{-1}(x)\right)=1\right\} .
$$


By Lemma 3.2(ii), $Y_{1}$ is contains some definable set $Y$ with $\lg \operatorname{dim}(Y)=\lg \operatorname{dim}(X)-$ 1. Now, $X_{l}$ is contained in the union of the following two sets:

$$
\begin{aligned}
& A_{1}=\left\{x \in r(Y): \operatorname{lgdim}\left(r^{-1}(x)\right) \geq l-1\right\} \text { and } \\
& B_{1}=\left\{x \in r(q(X) \backslash Y): \operatorname{lgdim}\left(r^{-1}(x)\right)=l\right\} .
\end{aligned}
$$

By the Inductive Hypothesis, $A_{1}$ is contained in a definable set $A$ with

$$
\lg \operatorname{dim}(A)=\lg \operatorname{dim}(Y)-(l-1)=\lg \operatorname{dim}(X)-l
$$

and $B_{1}$ is contained in a definable set $B$ with

$$
\lg \operatorname{dim}(B)=\lg \operatorname{dim}(q(X) \backslash Y)-l \leq \lg \operatorname{dim}(X)-l .
$$

Hence $X_{l}$ is contained in the definable set $Z_{l}=A \cup B$, satisfying $\operatorname{lgdim}\left(Z_{l}\right) \leq$ $\lg \operatorname{dim}(X)-l$. By Lemma 3.2(ii), $\lg \operatorname{dim}\left(Z_{l}\right)=\lg \operatorname{dim}(X)-l$.

We are finally in the position to prove the promised corollary on the definability of long dimension. Note that this corollary is not needed in the rest of the paper, but it is recorded here in the interests of completeness.

Corollary 3.4. Let $X \subseteq M^{n+m}$ be a definable set and denote by $\pi: M^{n+m} \rightarrow M^{n}$ the usual projection. For $0 \leq l \leq m$, let

$$
l(X)=\left\{x \in \pi(X): \pi^{-1}(x) \text { has long dimension } l\right\} .
$$

Then there are definable subsets $Y, Z \subseteq \pi(X)$ with $Y \subseteq l(X) \subseteq Z$ such that

$$
\lg \operatorname{dim}(Y)=\lg \operatorname{dim}(Z)=\lg \operatorname{dim}(X)-l .
$$

Proof. With the notation of the previous two lemmas, let $Y=Y_{l} \backslash Z_{l+1}$ and $Z=$ $Z_{l+1}$. Since $\operatorname{lgdim}\left(Y_{l}\right)=\max \left\{\operatorname{lgdim}(Y), \operatorname{lgdim}\left(Z_{l+1}\right)\right\}$, it follows that $\lg \operatorname{dim}(Y)$ is as desired (and, clearly, so is $\lg \operatorname{dim}(Z)$ ).

\section{Pregeometries}

In this section we develop the combinatorial counterpart of the long dimension and define the corresponding notion of 'long-genericity'. This notion is used in the application to definable groups in the next section.

Definition 4.1. A (finitary) pregeometry is a pair $(S, c l)$, where $S$ is a set and $c l: P(S) \rightarrow P(S)$ is a closure operator satisfying, for all $A, B \subseteq S$ and $a, b \in S$ :

(i) $A \subseteq \operatorname{cl}(A)$

(ii) $A \subseteq B \Rightarrow \operatorname{cl}(A) \subseteq \operatorname{cl}(B)$

(iii) $\operatorname{cl}(\operatorname{cl}(A))=\operatorname{cl}(A)$

(iv) $\operatorname{cl}(A)=\cup\{\operatorname{cl}(B): B \subseteq A$ finite $\}$

(v) (Exchange) $a \in \operatorname{cl}(b A) \backslash \operatorname{cl}(A) \Rightarrow b \in \operatorname{cl}(a A)$.

Definition 4.2. We define the short closure operator scl $: P(M) \rightarrow P(M)$ as:

$$
\begin{aligned}
& \operatorname{scl}(A)=\{a \in M: \text { there are } \bar{b} \subseteq A \text { and } \phi(x, \bar{y}) \text { from } \mathcal{L}, \text { such that } \\
&\phi(\mathcal{M}, \bar{b}) \text { is a short interval and } \mathcal{M} \vDash \phi(a, \bar{b})\} .
\end{aligned}
$$

We say that the formula $\phi(x, \bar{y}) \in \mathcal{L}$ witnesses $a \in \operatorname{scl}(\bar{b})$ if $\phi(\mathcal{M}, \bar{b})$ is a short interval and $\mathcal{M} \vDash \phi(a, \bar{b})$.

We will omit, as usually, the bar from tuples. 
Remark 4.3. Given a formula $\phi(x, y) \in \mathcal{L}$ witnessing $a \in \operatorname{scl}(b)$, we can form a formula $S_{a, b}^{\phi}(x, y) \in \mathcal{L}$ which is satisfied by the pair $(a, b)$ and such that for every $b^{\prime} \in M^{n}, S_{a, b}^{\phi}\left(\mathcal{M}, b^{\prime}\right)$ is short. Indeed, let $\kappa \in M$ be short such that

$$
\forall z_{1}, z_{2}\left[\phi\left(z_{1}, b\right) \wedge \phi\left(z_{2}, b\right) \rightarrow\left|z_{1}-z_{2}\right|<\kappa\right] .
$$

By [Pet3, Corollary 3.7(3)], $\kappa$ may be taken in $d c l(\emptyset)$. We then let

$$
S_{a, b}^{\phi}(x, y): \phi(x, y) \wedge \forall z_{1}, z_{2}\left[\phi\left(z_{1}, y\right) \wedge \phi\left(z_{2}, y\right) \rightarrow\left|z_{1}-z_{2}\right|<\kappa\right] .
$$

Lemma 4.4. $a \in \operatorname{scl}(b) \Leftrightarrow \exists a^{\prime} \in \operatorname{dcl}(b), a-a^{\prime}$ is short.

Proof. $(\Rightarrow)$. Let $f$ be a $\emptyset$-definable Skolem function for $S_{a, b}^{\phi}(x, y)$, where $\phi$ witnesses $a \in \operatorname{scl}(b)$; that is, for every $c \in M, \models \exists z S_{a, b}^{\phi}(z, c) \rightarrow S_{a, b}^{\phi}(f(c), c)$. Let $a^{\prime}=f(b)$.

$(\Leftarrow)$. Assume $\phi(x, y)$ witnesses $a^{\prime} \in d c l(b)$. Let $\kappa \in d c l(\emptyset)$ such that $\left|a-a^{\prime}\right|<\kappa$. Then $a$ satisfies the following short formula:

$$
\exists x^{\prime} \phi\left(x^{\prime}, b\right) \wedge\left(\left|x-x^{\prime}\right|<\kappa\right) .
$$

Lemma 4.5. $(M, s c l)$ is a pregeometry.

Proof. Properties (i), (ii) and (iv) are straightforward.

(iii). This boils down to the fact that (Lemma 3.2(ii)) a short union of short sets is short. We provide the details. Let $a \in \operatorname{scl}(\bar{b})$, where $\bar{b}=\left(b_{1}, \ldots, b_{n}\right) \in M^{n}$, such that each $b_{i} \in \operatorname{scl}(\bar{c})$, for some $\bar{c} \subseteq A$. Assume that $\psi(x, \bar{b})$ witnesses $a \in \operatorname{scl}(\bar{b})$, and that for each $i=1, \ldots, n, \phi_{i}\left(y_{i}, \bar{c}\right)$ witnesses $b_{i} \in \operatorname{scl}(\bar{c})$, where $\psi, \phi_{i} \in \mathcal{L}$. Denote

$$
S(\bar{y}, \bar{z}):=S_{b_{1}, \bar{c}}^{\phi_{1}}\left(y_{1}, \bar{z}\right) \wedge \cdots \wedge S_{b_{n}, \bar{c}}^{\phi_{n}}\left(y_{n}, \bar{z}\right)
$$

Then the set $X$ defined by the formula

$$
\exists \bar{y} S(\bar{y}, \bar{c}) \wedge S_{a, \bar{b}}^{\psi}(x, \bar{y})
$$

is $\bar{c}$-definable and contains $a$. We show that $X$ is short. Clearly, the set

$$
X^{\prime}=\bigcup_{\bar{y} \in S(\mathcal{M}, \bar{c})}\{\bar{y}\} \times S_{a, \bar{b}}^{\psi}(\mathcal{M}, \bar{y})
$$

has long dimension at least the long dimension of $X$, since the function $f:(\bar{y}, x) \mapsto$ $x$ maps $X^{\prime}$ onto $X$. But $X^{\prime}$ is a short union of short sets and, by Lemma 3.2(ii), it must have long dimension 0 .

(v). Without loss of generality, assume $A=\emptyset$. Let $\phi(x, y)$ be a formula witnessing $a \in \operatorname{scl}(b)$. We assume that $b \notin \operatorname{scl}(a)$ and show $a \in \operatorname{scl}(\emptyset)$. Let $f(x)$ be a $\emptyset$-definable Skolem function for $S_{a, b}^{\phi}(x, y)$. Let $\kappa \in M$ be short and in $d \operatorname{cl}(\emptyset)$ such that

$$
\forall z_{1}, z_{2}\left[\phi\left(z_{1}, b\right) \wedge \phi\left(z_{2}, b\right) \rightarrow\left|z_{1}-z_{2}\right|<\kappa\right] .
$$

(see Remark 4.3). Let

$$
Y=\left\{b^{\prime} \in M:\left|f\left(b^{\prime}\right)-a\right|<\kappa\right\} .
$$

Then since $Y$ is $a$-definable and contains $b$, it must be long. By Lemma 2.1, there is some interval contained in $Y$ on which $f$ is constant, equal say to $a^{\prime}$. But then $a^{\prime} \in d c l(\emptyset)$ and, by Lemma 4.4, $a \in \operatorname{scl}(\emptyset)$. 
Definition 4.6. Let $A, B \subseteq M$. We say that $B$ is scl-independent over $A$ if for all $b \in B, b \notin \operatorname{scl}(A \cup(B \backslash\{b\}))$. A maximal scl-independent subset of $B$ over $A$ is called a basis for $B$ over $A$.

By the Exchange property in a pregeometric theory, any two bases for $B$ over $A$ have the same cardinality. This allows us to define the rank of $B$ over $A$ :

$$
\operatorname{rank}(B / A)=\text { the cardinality of any basis of } B \text { over } A \text {. }
$$

Lemma 4.7. If $p$ is a partial type over $A \subseteq M$ and $a \models p$ with $\operatorname{rank}(a)=m$, then for any set $B \supseteq A$ there is $a^{\prime} \models p$ (possibly in an elementary extension) such that $\operatorname{rank}\left(a^{\prime}\right) \geq m$.

Proof. The proof of the analogous result for the usual rank (coming from $a c l$ ) is given, for example, in [G, page 315]. The proof of the present lemma is word-byword the same with that one, after replacing an 'algebraic formula' by a 'short formula' in the definition of $\Phi_{B}^{m}$ ([G, Definition 2.2]) and the notion of 'algebraic independence' by that of 'scl-independence' we have here.

Definition 4.8. Let $p$ be a partial type over $A \subset M$. The short closure dimension of $p$ is defined as follows:

$$
\operatorname{scl}-\operatorname{dim}(p)=\max \{\operatorname{rank}(\bar{a} / A): \bar{a} \subset M \text { and } \bar{a} \models p\} .
$$

Let $X$ be a definable set. Then the short closure dimension of $X$, denoted by $\operatorname{scl}-\operatorname{dim}(X)$ is the dimension of its defining formula.

In Corollary 4.10 below we establish that the cl-dimension of a definable set coincides with its long dimension we defined earlier. We note that the equivalence between the usual geometric and topological dimension was proved in [Pi1].

Lemma 4.9. Let $\bar{a} \subseteq M$ be an $n$-tuple and $A \subseteq M$ a set. Then $\operatorname{rank}(a / A)=n$ if and only if $\bar{a}$ does not belong to any $A$-definable set with long dimension $<n$.

Proof. $(\Leftarrow)$ Assume $\bar{a}=\left(a_{1}, \ldots, a_{n}\right)$ and $\operatorname{rank}(\bar{a} / A)<n$. Then for some $i$, say $i=1, a_{1} \in \operatorname{scl}\left(A \cup\left\{a_{2}, \ldots, a_{n}\right\}\right)$. Let $\phi(x, \bar{y})$ be an $\mathcal{L}(A)$-formula witnessing this fact. Recall from Remark 4.3 that the $\mathcal{L}(A)$-formula $S_{\bar{a}}^{\phi}(x, \bar{y})$ is satisfied by $\bar{a}$ and for every $b^{\prime} \in M^{n-1}, S_{\bar{a}}^{\phi}\left(\mathcal{M}, b^{\prime}\right)$ is short. By Lemma 3.2(ii), $S_{\bar{a}}^{\phi}\left(\mathcal{M}^{n}\right)$ has long dimension $<n$.

$(\Rightarrow)$ We prove the statement by induction on $n$. For $n=1$, it is clear. Suppose it is proved for $n$. Let $\bar{a}=\left(a_{1}, \ldots, a_{n+1}\right)$ be a tuple of rank, over $A$, equal to $n+1$ and assume, for a contradiction, that $X$ is an $A$-definable set containing $a$ with $\lg \operatorname{dim}(X)<n+1$. By cell decomposition, we may assume that $X$ is an $A$-definable cell. If $X$ is the graph of a function, then $a_{n+1}$ is in $d c l\left(A \cup\left\{a_{1}, \ldots, a_{n}\right\}\right)$ and hence in $\operatorname{scl}\left(A \cup\left\{a_{1}, \ldots, a_{n}\right\}\right)$, a contradiction. Now assume that $X$ is a cylinder. By the Refined Structure Theorem, we may assume that $X=(f, g)_{\pi(X)}$ is a cylinder whose domain is an $A$-definable long cone such that $f$ and $g$ are almost linear with respect to it. Since $\operatorname{rank}(\bar{a} / A)=n+1, g\left(a_{1}, \ldots, a_{n}\right)-f\left(a_{1}, \ldots, a_{n}\right)$ must be long. But by Inductive Hypothesis, $\lg \operatorname{dim}(\pi(X))=n$. Hence, by Lemma $2.22, \lg \operatorname{dim}(X)=n+1$, a contradiction.

Corollary 4.10. For every definable $X \subseteq M^{n}$,

$$
\lg \operatorname{dim}(X)=\operatorname{scl}-\operatorname{dim}(X)
$$


Proof. By Lemma 4.9 and the (easy) fact that every $A$-definable $k$-long cone contains a tuple $a$ with $\operatorname{rank}(a / A)=k$.

4.1. Long-generics. For a treatment of the classical notion of generic elements, corresponding to the algebraic closure $a c l$, see [Pi2]. Here we introduce the corresponding notion for $s c l$.

Definition 4.11. Let $X \subseteq M^{n}$ be an $A$-definable set, and let $\bar{a} \in X$. We say that $\bar{a}$ is a long-generic element of $X$ over $A$ if it does not belong to any $A$-definable set of long dimension $<\operatorname{lgdim}(X)$. If $A=\emptyset$, we call $\bar{a}$ a long-generic element of $X$.

In a sufficiently saturated o-minimal structure, long-generic elements always exist. More precisely, every $A$-definable set $X$ contains a long-generic element over $A$. Indeed, by Compactness and Lemma 2.21(v), the collection of all formulas which express that $x$ belongs to $X$ but not to any $A$-definable set of long dimension $<\lg \operatorname{dim}(X)$ is consistent.

A definable subset $V$ of a definable set $X$ is called long-large in $X$ if $\lg \operatorname{dim}(X \backslash$ $V)<\operatorname{lgdim}(X)$. In a sufficiently saturated o-minimal structure, $V$ is long-large in $X$ if and only if for every $A$ over which $V$ and $X$ are defined, $V$ contains every long-generic element $a$ in $X$ over $A$.

Two long-generics are called independent if one (each) of them is long-generic over the other.

Let $G$ be a definable abelian group. Let us recall the notion of a left generic set (not to be confused with the notion of a generic element). A subset $X \subseteq G$ is called left n-generic if $n$ left translates of $X$ cover $G$. It is called left generic if it is left $n$-generic for some $n$. We recall from [ElSt, Lemma 3.10] (see [PeS] for the notion of definable compactness):

Fact 4.12 (Generic Lemma). Assume $G$ is definably compact. Then, for every definable subset $X \subseteq G$, either $X$ or $G \backslash X$ is left generic.

The facts that $(M, s c l)$ is a pregeometry and that the scl-dim agrees with lgdim imply:

Claim 4.13. Let $G=\langle G, \cdot\rangle$ be a definable group with $\lg \operatorname{dim}(G)=n$. Then

(1) If $X \subseteq G$ long-large in $G$, then $X$ is left $(n+1)$-generic in $G$.

(2) If $a$ and $g \in G$ are independent long-generics, then so are $a$ and $a \cdot g$.

Proof. The proof is standard. (1) is word-by-word the same with that of [Pet2, Fact 5.2] after replacing: a) the notion of a 'large' set by that of a 'long-large' set, b) the 'dimension' of a definable set by 'long dimension', and c) the 'dimension' of a tuple by 'rank'. (2) is straightforward using the Exchange property.

Note that none of the notions 'generic element' and 'long-generic element' implies the other.

Lemma 4.14. Let $X, W$ be definable subsets of a definable group $G$. Assume that $X$ is a long-large subset of $W$ and that $W$ is left generic in $G$. Then $X$ is left generic in $G$.

Proof. This is similar to the proof of [PePi, Lemma 3.4(ii)]. Since $W$ is left generic we can write $G=g_{1} W \cup \cdots \cup g_{m} W$. Let $Y=W \backslash X$. Then $Z=g_{1} Y \cup \cdots \cup g_{m} Y$ has long dimension $<\lg \operatorname{dim}(G)$. So, by Claim 4.13, finitely many left translates of $G \backslash Z$ cover $G$. It follows then that finitely many left translates of $X$ cover $G$. 
We record one more lemma, which however will not be used in this paper:

Lemma 4.15. Let $G$ be a definable group and $X$ a definable subset of $G$. If $X$ is left generic in $G$ then $\operatorname{lgdim}(X)=\operatorname{lgdim}(G)$.

Proof. Since the group conjugation is a definable bijection, the statement follows from Lemma 2.21(v) and Corollary 2.26.

\section{The LOCAL STRUCTURE OF SEMI-BOUNDED GROUPS}

We fix a $\emptyset$-definable group $G=\left\langle G, \oplus, e_{G}\right\rangle$, with dimension $n$ and long dimension $k$.

By [Pi2], we know that every group definable in an o-minimal structure can be equipped with a unique definable manifold topology that makes it into a topological group, called the $t$-topology. We refer the reader elsewhere for the basic facts about the $t$-topology (which we will not make any essential use of, anyway). By [EdEl, Fact 2.3], we may assume that $G \subseteq M^{n}$.

Remark 5.1. By the Refined Structure Theorem, Lemma 2.20 and Corollary 4.10, for any two independent long-generic elements $a$ and $b$ of $G$ and any $\emptyset$-definable function $f: G \times G \rightarrow G$, there are $k$-long cones $C_{a}$ and $C_{b}$ in $G$ containing $a$ and $b$, respectively, such that for all $x \in C_{a}$ and $y \in C_{b}$,

$$
f(x, y)=\lambda x+\mu y+d,
$$

for some fixed $\lambda, \mu \in \mathbb{M}(n, \Lambda)$ and $d \in M^{n}$. Moreover, $\lambda$ and $\mu$ have to be invertible matrices (for example, setting $y=b, x \oplus b=\lambda x+\mu b+d$ is invertible, showing that $\lambda$ is invertible).

Lemma 5.2. For every two independent long-generics $a, b \in G$, there is a $k$-long cone $C_{a}$ containing a, invertible $\lambda, \lambda^{\prime} \in \mathbb{M}(n, \Lambda)$ and $c, c^{\prime} \in M^{n}$, such that for all $x \in C_{a}$,

$$
x \ominus a \oplus b=\lambda x+c \text { and } \ominus a \oplus b \oplus x=\lambda^{\prime} x+c^{\prime} .
$$

Proof. By Claim 4.13, since $a$ and $b$ are independent long-generics of $G, a$ and $\ominus a \oplus b$ are independent long-generics of $G$ as well. Therefore, by Remark 5.1, there are cones $C_{a}$ of $a$ and $C_{\ominus a \oplus b}$ of $\ominus a \oplus b$ in $G$, as well as invertible $\lambda, \mu \in \mathbb{M}(n, D)$ and $d \in M^{n}$, such that $\forall x \in C_{a}, \forall y \in C_{\ominus a \oplus b}$,

$$
x \oplus y=\lambda x+\mu y+d .
$$

In particular, for all $x \in C_{a}, x \ominus a \oplus b=\lambda x+\mu(\ominus a \oplus b)+d$. Letting $c=\mu(\ominus a \oplus b)+d$ shows the first equality. The second equality can be shown similarly.

We are now ready to prove the local theorem for semi-bounded groups.

Theorem 5.3. Let a be a long-generic element of $G$. Then there is a k-long cone $C_{a} \subseteq G$, such that for every $x, y \in C_{a}$,

$$
x \ominus a \oplus y=x-a+y .
$$

Proof. We first prove:

Claim. There is a $k$-long cone $C_{a} \subseteq G$ containing a, and $\lambda, \mu \in \mathbb{M}(n, \Lambda)$ and $d \in M^{n}$, such that for all $x, y \in C_{a}$,

$$
x \ominus a \oplus y=\lambda x+\mu y+d .
$$


Proof of the Claim. Let $a_{1}$ be a long-generic element of $G$ independent from $a$. Then $a_{2}=a \ominus a_{1}$ is also a long-generic element of $G$ independent from $a$. By Lemma 5.2 , we can find $k$-long cones $C$ and $C^{\prime}$ in $G$ containing $a$, and neighborhoods $V_{a_{1}}$ of $a_{1}$ and $V_{a_{2}}$ of $a_{2}$ in $G$, as well as $\lambda_{1}, \lambda_{2} \in \mathbb{M}(n, \Lambda)$ and $c_{1}, c_{2} \in M^{n}$, such that $\forall x \in C, \forall y \in C^{\prime}$ :

$$
x \ominus a_{2}=\lambda_{1} x+c_{1} \in V_{a_{1}} \text { and } \ominus a_{1} \oplus y=\lambda_{2} y+c_{2} \in V_{a_{2}} .
$$

Since $a$ is long-generic, it must be contained in a $k$-long cone $C_{a} \subseteq C \cap C^{\prime}$, on which, of course, every $x$ and $y$ satisfy the above equations.

Now, by Remark 5.1 and since $a_{1}$ and $a_{2}=a \ominus a_{1}$ are independent long-generics of $G$, there are $k$-long cones $C_{a_{1}}$ and $C_{a_{2}}$ containing $a_{1}$ and $a_{2}$, respectively, such that for some fixed $\nu, \xi \in \mathbb{M}(n, \Lambda)$ and $o \in M^{n}$, we have: $\forall x \in C_{a_{1}}, \forall y \in C_{a_{2}}, x \oplus y=$ $\nu x+\xi y+\varepsilon$. By continuity of $\oplus$, we could choose $C_{a}$ so that $V_{a_{1}} \subseteq C_{a_{1}}$ and $V_{a_{2}} \subseteq C_{a_{2}}$. Then for all $x, y \in C_{a}$, we have:

$$
\begin{aligned}
x \ominus a \oplus y & =x \ominus a \oplus a_{1} \ominus a_{1} \oplus y \\
& =\left(x \ominus a_{2}\right) \oplus\left(\ominus a_{1} \oplus y\right) \\
& =\nu\left(\lambda_{1} x+c_{1}\right)+\xi\left(\lambda_{2} y+c_{2}\right)+o \\
& =\nu \lambda_{1} x+\xi \lambda_{2} y+\nu c_{1}+\xi c_{2}+o
\end{aligned}
$$

Setting $\lambda=\nu \lambda_{1}, \mu=\xi \lambda_{2}$, and $d=\nu c_{1}+\xi c_{2}+o$ finishes the proof of the lemma.

By the Claim, for all $x, y \in C_{a}$,

$$
\begin{aligned}
& y=a \ominus a \oplus y=\lambda a+\mu y+d \\
& x=x \ominus a \oplus a=\lambda x+\mu a+d \\
& a=a \ominus a \oplus a=\lambda a+\mu a+d .
\end{aligned}
$$

Hence, $x-a+y=\lambda x+\mu y+d=x \ominus a \oplus y$.

We conclude with a stronger version of the local theorem that we expect to be useful in a future global analysis for semi-bounded groups. By [Pi2], we know that the $t$-topology of $G$ coincides with the subspace topology on a large open subset $W^{G}$. The proof of the following proposition involves all machinery developed so far.

Proposition 5.4. Assume $G$ is definably compact. There is a left generic $k$-long cone $C$ contained in $G$, on which the two topologies coincide, and for every $a \in C$, there is a relatively open subset $V_{a}$ of $a+\langle C\rangle$ containing a, such that $\forall x, y \in V_{a}$,

$$
x \ominus a \oplus y=x-a+y .
$$

Proof. By the Refined Structure Theorem, $W^{G}$ is the union of finitely many long cones $C_{1}, \ldots, C_{l}$. Let $\bar{v}_{j}=\left(v_{j 1}, \ldots, v_{j k_{j}}\right)$ be the direction of each $C_{j}$. By the Generic Lemma, one of the $C_{j}$ 's, say $C_{1}$, is a left generic $k$-long cone.

Every long-generic element $a$ is contained in some $k$-long cone contained in $G$ with direction some $\bar{v}_{j}$ on which (4) holds: indeed, by Theorem 5.3, $a$ is contained in some $k$-long cone $D$ on which (4) holds. Now, if $a$ is also contained in $C_{j}$, then, by Corollary 2.17 , it is not hard to see that some $k$-long cone with direction $\bar{v}_{j}$ must be contained in $D$ and contain $a$.

Consider now the following property, for an element $a \in C_{1}$ :

$\left.{ }^{*}\right)$ there is a relatively open subset $V_{a}$ of $a+\left\langle C_{1}\right\rangle$ containing $a$, such that $\forall x, y \in V_{a}$, (4) holds. 
The set $X$ of elements of $C_{1}$ that satisfy $(*)$ is clearly definable. We claim that it is also long-large in $C_{1}$.

Clearly, it suffices to prove that every long-generic element of $C_{1}$ satisfies (*). Let $a$ be a long-generic element of $C_{1}$. If $a$ belongs to a $k$-long cone of direction $\bar{v}_{1}$ on which (4) holds, then we are done. Hence, by the second paragraph above, it clearly suffices to show that for every $j \neq i$, the set $A_{j}$ of all elements of $C_{1}$ that belong to a $k$-long cone of direction $\bar{v}_{j}$ but do not satisfy $\left({ }^{*}\right)$, is contained in a definable set of long dimension $<k$. To see this, note that if $a \in A_{j}$, then by Corollaries 2.16 and 2.17 , one of the $v_{j 1}, \ldots, v_{j k_{j}}$, say $v_{j 1}$, must be so that for every $t \in M, v_{j 1} t \notin\left\langle C_{1}\right\rangle$. Let $\kappa$ be a tall element and $D_{j}=\left\{v_{j 1} t: t \in(0, \kappa)\right\}$. The set

$$
K_{j}=\left(C_{1}+D_{j}\right) \cap G
$$

has long dimension $\leq k$, as a subset of $G$. Hence, by Lemma 3.3, and since each $D_{j}$ has long dimension $1, A_{j}$ is contained in a definable set of long dimension $\leq \lg \operatorname{dim}\left(K_{j}\right)-1$.

We have proved that $X$ is long-large in $C_{1}$. By Lemma 4.14, $X$ is generic. By the Refined Structure Theorem, the Generic Lemma and the Lemma on Subcones, there is a generic $k$-long cone $C$ contained in $X$ with the desired property.

\section{Appendix}

Lemma 2.8 will follow from the following statement.

Lemma 6.1. Let $w_{1}, \ldots, w_{n}, w_{n+1}, \ldots, w_{n+k} \in \Lambda^{n}$ be $M$-independent and $\lambda_{1}, \ldots, \lambda_{n} \in$ $\Lambda^{n}$. Let $t_{1}, \ldots, t_{n} \in M$ be non-zero elements and, for every $i=1, \ldots, n$, let $r_{i}^{1}, \ldots, r_{i}^{k} \in M$ be such that:

$$
\begin{gathered}
w_{1} t_{1}+\sum_{j=1}^{k} w_{n+j} r_{1}^{j}=\lambda_{1} s_{1}^{1}+\cdots+\lambda_{n} s_{1}^{n} \\
\vdots \\
w_{n} t_{n}+\sum_{j=1}^{k} w_{n+j} r_{n}^{j}=\lambda_{1} s_{n}^{1}+\cdots+\lambda_{n} s_{n}^{n}
\end{gathered}
$$

for some $s_{i}^{j} \in M$. Then there non-zero $a_{1}, \ldots, a_{n} \in M$ and $b_{i}^{j} \in M, i=1, \ldots, n$, $j=1, \ldots, n+k$, such that:

$$
\begin{aligned}
\lambda_{1} a_{1} & =w_{1} b_{1}^{1}+\cdots+w_{n+k} b_{1}^{n+k} \\
& \vdots \\
\lambda_{n} a_{n} & =w_{1} b_{n}^{1}+\cdots+w_{n+k} b_{n}^{n+k}
\end{aligned}
$$

Proof. By induction on $n$. For $n=1$, it is trivial. Assume $n>1$ and that we know the statement for $<n$ equations. Since $w_{1}, \ldots, w_{n+k} \in \Lambda^{n}$ are $M$-independent and $t_{1} \neq 0, w_{1} t_{1}+\sum_{j=1}^{k} w_{n+j} r_{1}^{j} \neq 0$. Hence there is some $s_{1}^{j}$, say $s_{1}^{1}$, which is not zero. Since

$$
\lambda_{1} s_{1}^{1}=w_{1} t_{1}+\sum_{j=1}^{k} w_{n+j} r_{1}^{j}-\left(\lambda_{2} s_{1}^{2}+\cdots+\lambda_{n} s_{1}^{n}\right)
$$


Lemma 2.7 gives, for every $i=2, \ldots, n$,

$$
\lambda_{1} s_{i}^{1}=w_{1} T_{i}+\sum_{j=1}^{k} w_{n+j} R_{i}^{j}-\left(\lambda_{2} S_{i}^{2}+\cdots+\lambda_{n} S_{i}^{n}\right)
$$

for some $S_{i}^{2}, \ldots, S_{i}^{n}, T_{i}, R_{i}^{1}, \ldots R_{i}^{k} \in M$. By substituting into the original system of equations, we obtain:

$$
\begin{gathered}
w_{2} t_{2}-w_{1} T_{1}+\sum_{j=1}^{k} w_{n+j}\left(r_{2}^{j}-R_{2}^{j}\right)=\lambda_{2}\left(s_{2}^{2}-S_{2}^{2}\right)+\cdots+\lambda_{n}\left(s_{2}^{n}-S_{2}^{n}\right) \\
\vdots \\
w_{n} t_{n}-w_{1} T_{1}+\sum_{j=1}^{k} w_{n+j}\left(r_{n}^{j}-R_{n}^{j}\right)=\lambda_{2}\left(s_{n}^{2}-S_{n}^{2}\right)+\cdots+\lambda_{n}\left(s_{n}^{n}-S_{n}^{n}\right)
\end{gathered}
$$

Now apply the Inductive Hypothesis, use Lemma 2.7 to solve for each of $\lambda_{2} s_{1}^{2}, \ldots, \lambda_{n} s_{1}^{n}$ in terms of $w_{1}, \ldots, w_{n+k}$ and, finally, substitute into (5).

\section{REFERENCES}

[Bel] O. Belegradek, Semi-bounded relations in ordered modules, J. of Symbolic Logic 69, n. 2 (2004), 499-517.

[DK] H. Delfs and M. Knebusch, Locally Semi-Algebraic Spaces, Lecture Notes in Mathematics, Springer-Verlag, Berlin, 1985.

[vdD1] L. van den Dries, Dense pairs of o-minimal structures, Fundamenta Mathematicae 157 (1988), 61-78.

[vdD2] L. van den Dries, Tame topology and o-minimal structures, Cambridge University Press, Cambridge, 1998.

[Ed] M. Edmundo, Structure theorems for o-minimal expansions of groups, Ann. Pure Appl. Logic 102 (2000), 159-181.

[EdEl] M. Edmundo and P. Eleftheriou, Definable group extensions in semi-bounded o-minimal structures, Math. Logic Quarterly, Volume 55 (2009), 598-604.

[El] P. Eleftheriou, Compact domination for groups definable in linear o-minimal structures, Archive for Mathematical Logic, Volume 48, Number 7 (2009), 607-623.

[ElSt] P. Eleftheriou and S. Starchenko, Groups definable in ordered vector spaces over ordered division rings, The Journal of Symbolic Logic 72 (2007), 1108-1140.

[G] J. Gagelman, Stability in geometric theories, Annals of Pure and Applied Logic 132 (2005), 313-326.

[HL] E. Hrushovski and F. Loeser, Non-archimedean tame topology and stably dominated types, Preprint (2010).

[HP] E. Hrushovski and A. Pillay, On NIP and invariant measures, Preprint (2009).

[HPP] E. Hrushovski, Y. Peterzil and A. Pillay, Groups, measures, and the NIP, J. Amer. Math. Soc. 21 (2008), 563-596.

[LP] J. Loveys and Y. Peterzil, Linear o-minimal structures, Israel Journal of Mathematics 81 (1993), 1-30.

[Ot] M. Otero A survey on groups definable in o-minimal structures, in Model Theory with Applications to Algebra and Analysis, vol. 2, Editors: Z. Chatzidakis, D. Macpherson, A. Pillay and A. Wilkie, LMS LNS 350, Cambridge Univ. Press (2008), 177-206.

[Pet1] Y. Peterzil, A structure theorem for semibounded sets in the reals, The Journal of Symbolic Logic 57 (1992), 779-794.

[Pet2] Y. Peterzil, Pillay's conjecture and its solution - a survey, Proceedings of the Wroclaw Logic Colloquium (2007).

[Pet3] Y. Peterzil, Returning to semi-bounded sets, J. Symbolic Logic 74 (2009), 597-617.

$[\mathrm{PePi}] \quad$ Y. Peterzil and A. Pillay, Generic sets in definably compact groups, Fund. Math. 193 (2007), 153-170 
[PeSt] Y. Peterzil and S. Starchenko, A trichotomy theorem for o-minimal structures, Proceedings of London Math. Soc. 77 (1998), no. 3, 481-523.

[PeS] Y. Peterzil and C. Steinhorn, Definable compactness and definable subgroups of ominimal groups, J. London Math. Soc. (2) 69 (1999), 769-786.

[Pi1] A. Pillay, Some remarks on definable equivalence relations in o-minimal structures, The Journal of Symbolic Logic, Volume 51, Number 3 (1986), 709-714.

[Pi2] A. Pillay, On groups and fields definable in o-minimal structures, J. Pure Appl. Algebra 53 (1988), 239-255.

[Pi3] A. Pillay, Type definability, compact Lie groups, and o-minimality, J. of Math. Logic, 4 (2004), 147-162.

CMaf, Universidade de Lisboa, Av. Prof. Gama Pinto 2, 1649-003 Lisboa, Portugal E-mail address: pelefthe@gmail.com 